\title{
Improve the Hole Size-dependent Refractive Index Sensitivity of Au-Ag Nanocages by Tuning the Alloy Composition
}

Jianjun LI ( $\nabla_{\text {jjunli@mail.xjtu.edu.cn ) }}$

Xi'an Jiaotong University https://orcid.org/0000-0002-1916-6313

Qiu-Xiang Qin

Xi'an Jiaotong University

Guo-Jun Weng

Xi'an Jiaotong University

Jian Zhu

Xi'an Jiaotong University

Jun-Wu Zhao

Xi'an Jiaotong University

\section{Research Article}

Keywords: Au-Ag alloy nanocages, Au-Ag ratio, Hole size, Plasmon coupling

Posted Date: May 19th, 2021

DOI: https://doi.org/10.21203/rs.3.rs-482206/v1

License: (c) (1) This work is licensed under a Creative Commons Attribution 4.0 International License. Read Full License

Version of Record: A version of this preprint was published at Plasmonics on October 7th, 2021. See the published version at https://doi.org/10.1007/s11468-021-01536-0. 


\title{
Improve the Hole Size-dependent Refractive Index Sensitivity of Au-Ag Nanocages by Tuning the Alloy Composition
}

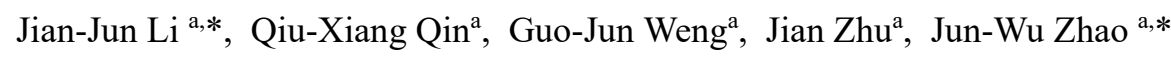

${ }^{a}$ The Key Laboratory of Biomedical Information Engineering of Ministry of Education, School of Life Science and Technology, Xi' an Jiaotong University, Xi' an 710049, China

* Authors to whom correspondence should be addressed.

Corresponding Author: Jian-Jun Li ; Jun-Wu Zhao

Electronic mail: jjunli@mail.xjtu.edu.cn (J.J. Li); nanoptzhao@163.com (J.W.Zhao)

Tel.: 86-29-82664224.

Address: School of Life Science and Technology,

Xi'an Jiaotong University, Xi'an, 710049, Peoples Republic of China

\begin{abstract}
In this study, $\mathrm{Au}-\mathrm{Ag}$ nanoboxes are converted into $\mathrm{Au}-\mathrm{Ag}$ alloy nanocages by increasing the hole size. The extinction spectrum and the refractive index sensing characteristics of $\mathrm{Au}-\mathrm{Ag}$ alloy nanocages with different geometric parameters are studied by using discrete dipole approximation method (DDA). With the increase of Au composition, the peak of local surface plasmon resonance (LSPR) shows approximately linear red shift and the sensitivity factor shows approximately linear decrease. The refractive index sensitivity can be effectively controlled by the Au-Ag ratio at large hole size because the hole and cavity surfaces distribute more environmental dielectric components. Therefore, increasing the hole size and decreasing the Au-Ag ratio can improve the refractive index sensitivity. In order to illustrate the influence of alloy composition on the LSPR characteristics and the refractive index sensitivity, the local electric field distributions under different geometric parameters is plotted. We find that the electric field direction on the hole and cavity surfaces are controlled by the Au-Ag ratio and environmental dielectric constant. Moreover, the field vectors on the hole and cavity surfaces are formed by the superposition of the incident field, the electric field generated by the oscillating electrons on the outer surface, and the polarized field in the environmental dielectric constant.
\end{abstract}

Keywords: $\mathrm{Au}-\mathrm{Ag}$ alloy nanocages, $\mathrm{Au}-\mathrm{Ag}$ ratio, Hole size, Plasmon coupling 


\section{Introduction}

In recent years, the investigation on the optical properties of noble metal nanoparticles of $\mathrm{Au}$ [1], $\mathrm{Ag}[2]$, and $\mathrm{Au}-\mathrm{Ag}$ alloy [3,4] have greatly promoted the development of noble metal nanoparticles in biochemical spectral sensing [5], imaging [6-8], and various biomedical applications. Under the action of the external incident light field, the surface free electrons in the noble metal nanoparticles will collectively oscillate at the same frequency as the incident electromagnetic wave, which is called LSPR [9]. The intensity and wavelength of LSPR peak of noble metal nanoparticles are closely related to the metal type, size, morphology and environmental dielectric constant $[10,11]$, which is the basis of LSPR sensing application, and also provides the possibility of designing and manufacturing ultra-sensitive LSPR refractive index sensors $[12,13]$.

The superior optical properties of $\mathrm{Au}$ and Ag nanoparticles have attracted many researchers' attention, so they have been widely studied. Among them, the LSPR effect of Au nanoparticles enables them to have enhanced absorption and scattering characteristics, so Au nanoparticles have become commonly used drugs for the diagnosis, treatment and imaging in the biomedical field. In addition, the good biocompatibility of Au nanoparticles and easy connectivity with various biomolecule ligands and antibodies, making them suitable for biosensing and detection $[14,15]$. Compared with Au nanoparticles, Ag nanoparticles have higher refractive index sensitivity, but their stability is poor under the same particle size and morphology [16]. With the development of society, the demand for nanomaterials with the advantages of these two metals is increasing day by day. The LSPR peak of Au nanoparticles and Ag nanoparticles are around $525 \mathrm{~nm}$ and $408 \mathrm{~nm}$, respectively [17]. Au-Ag alloy nanomaterials can adjust the wavelength of LSPR peak to near infrared region [18]. Therefore, alloy nanomaterials with high stability and high LSPR response are of great significance for the preparation of sensors.

Nowadays, $\mathrm{Au}$ nanoparticles and $\mathrm{Ag}$ nanoparticles with various morphologies have been prepared, such as rods [19], triangles [20], cones [21], stars [22], nanoshells [23], nanocubes [24], nanoboxes [4], nanoframes [25, 26], and hollow nanocages [4]. Each type of nanoparticle has rich and adjustable LSPR characteristics. Also, the theoretical calculations of the LSPR characteristics and refractive index sensitivity of nanoparticles with arbitrary shape are mature, which provide reliable theoretical guidance for the experiment and promote the experimental synthesis and 
application research. The sharp corners in noble metal nanocubes provide them with abundant LSPR bands and stronger local electric fields [27], and more and more researchers have studied their optical properties in depth $[1,28,29]$. For example, when the size of the Ag nanocubes changes, the number of LSPR peaks in the spectrum increases and the position of LSPR peaks changes significantly [18]. Besides, compared with the Ag nanocubes, the LSPR peaks of Au nanocages [30], Au-Ag alloy nanoboxes [3] and Au-Ag alloy nanocages $[4,31]$ prepared by the galvanic substitution reaction [32] between the $\mathrm{Ag}$ nanocubes and $\mathrm{HAuCl}_{4}$ are redshifted, and the refractive index sensitivity [1] of the nanocages is higher than that of the nanoboxes [33] and the nanocubes [14]. At different stages of galvanic substitution, $\mathrm{Au}-\mathrm{Ag}$ ratio, hole size, wall thickness, and wall length $[34,35]$ are all important factors affecting the LSPR peak of nanocages, and the refractive index sensitivity of nanoparticles is related to the LSPR offset $[12,36]$. In fact, theoretical calculations have proved the influence of the Au-Ag ratio, wall thickness, wall length, environmental dielectric constant [10,11], and hole size on LSPR peak. The report of Sekhon [14] showed that the changes of alloy composition affected the optical properties of Au-Ag alloy nanocubes, and the alloy composition could change the sensitivity factor. Mahmoud [35] found that the peak wavelength of LSPR of Au nanocages and Au nanoframes blueshifted with the increase of the wall thickness. Similarly, the sensitivity factor of the Au nanoframes had a strong correlation with the aspect ratio, but had little dependence on the wall length [34]. Meanwhile, the sensitivity factor increases linearly with the increase of the aspect ratio (wall length/wall thickness). Zhu [1] also studied the influence of the hole size of the Au nanocages on the LSPR peak and refractive index sensitivity. They found that the LSPR peak wavelength and the refractive index sensitivity of $\mathrm{Au}$ nanocages increased exponentially with the increase of hole size. In addition, the Au-Ag alloy nanocages have adjustable wall holes, and the Au-Ag alloy nanocages were developed and designed as biochemical sensors for the detection of mercury ions [4].

On the other hand, the sensitivity of noble metal nanoparticles LSPR to the environmental dielectric constant has opened up a new field for LSPR sensing. As we all know, the position of LSPR peak depends on the environmental dielectric constant and the morphology, composition and structure of nanoparticles. In addition, the refractive index sensitivity is defined as the displacement momentum of the LSPR peak position caused by the change of unit refractive index. Therefore, the 
refractive index sensitivity factor depends on the morphology, size, structure and type of nanoparticles. Nanopolyhedron with large surface area and sharp tip has higher refractive index sensitivity factor. Therefore, people began to pay attention to the application of nanopolyhedron in refractive index sensors. In the study of refractive index sensitivity of Au nanotubes reported by Zhu et al, the LSPR optical response was calculated by adjusting the wall thickness and the environmental dielectric constant and it was found that the reduction of the environmental dielectric constant and the reduction of the wall thickness were optimized refractive index sensitivity [10]. Similarly, in Zhu' other research, calculation results based on a quasi-static model showed that the sensitivity of coaxial cable-type Au nanotubes increased with the increase of the inner diameter or total radius [11]. Sekhon et al. studied the sensing performance of Au-Ag alloy nanocubes by changing the alloy composition to adjust the LSPR band rang [14]. The results of Liaw et al. showed that the LSPR offset in Au-Ag alloy nanocages depends on the porosity. The greater the porosity, the greater the LSPR redshift [15]. The results of this series of studies show that these geometric parameters make plasmons have a wider spectral band range, thus obtaining sensing characteristics that are more sensitive to the geometric parameters. Interestingly, in the Au-Ag alloy nanocages [15], the tunable hole size and $\mathrm{Au}-\mathrm{Ag}$ ratio and the large inner and outer surface areas increase the tunability of LSPR in a wide spectral band, and make the plasmon coupling modes more complex. According to theoretical calculations, it has been confirmed that the porosity increase of the Au-Ag alloy nanocages and the increase of the environmental dielectric constant cause the redshift of the plasmon resonance peak [14], which means that the porosity may optimize the refractive index sensitivity of the Au-Ag alloy nanocages. Therefore, the search for the optimal geometric parameters of $\mathrm{Au}-\mathrm{Ag}$ alloy nanocages is crucial for the refractive index sensitivity. The effect of alloy composition on the LSPR peak of the Au-Ag alloy nanocages is unknown at present. How does the alloy composition affect the LSPR peak of the Au-Ag alloy nanocages with different hole sizes? What is the effect of alloy composition proportion on refractive index sensitivity? The influence of alloy composition on refractive index sensing performance has not been studied systematically.

The extinction spectrum and refractive index sensitivities of Au-Ag alloy nanocages with square hole size on the $\{100\}$ plane at different hole sizes and Au-Ag ratio are calculated by DDA theory. Combining the advantages of alloy nanomaterials and nanocages, we have studied the 
influence of many factors on refractive index sensitivity. The refractive index sensitivity of $\mathrm{Au}-\mathrm{Ag}$ alloy nanocages is related to LSPR offset. In this paper, the sensitivity factor is optimized by adjusting the Au-Ag ratio and the hole size. Besides, to clarify the influence of the environmental dielectric constant on the local electric field and LSPR characteristics of Au-Ag alloy nanocages. The physical mechanism of plasmon resonance peak characteristics affected by Au-Ag ratio, hole size, and environmental dielectric constant is discussed in detail, and the influence of these parameters on plasmon coupling is analyzed.

\section{The model and procedure}

\section{The DDA theory}

The theoretical numerical calculation of noble metal nanoparticles is mainly to investigate the effect of geometric parameters on plasmon resonance. To obtain the optical response of LSPR, we need to discuss the electromagnetic interaction between the incident light and nanoparticles under the framework of Maxwell's equations, but Maxwell's equations are limited to simple spheres [37] and ellipsoids. To be able to calculate the optical properties of noble metal nanoparticles of any shape, in 1964, Devoe first proposed the basic idea of DDA to solve any geometric shape by approximation method [38], but this method is limited to the aggregation of smaller wavelengths. In 1973, Purcell and Pennypacke modified and improved the DDA idea, and applied DDA to the calculation of optical properties of various particles [39]. Simply put, the DDA algorithm is to simulate the target shape with a finite number of dipole points, and the interaction of the dipole points after applying external electromagnetic light waves simulates the collective oscillation of free electrons. Then, under the given conditions, the optical response of the target shape to the geometric parameters is calculated [40]. Since the DDA idea was proposed, the geometric parameters, simulation accuracy and convergence, application fields, and program codes in the DDA method have been modified and improved to varying degrees [40-47]. DDA has become a powerful theoretical method that can simulate nanoparticles of any shape. In this paper, DDSCAT.7.3 software is used to simulate the plasma optical response and electric field distribution of Au-Ag alloy nanocages under different geometric parameters. In the Au-Ag alloy nanocages model in this article, the origin of the coordinates is at the center of the model, and the distance between the dipole points 
is set to $1 \mathrm{~nm}$. Besides, the accuracy and convergence of DDA calculations are closely related to the number of dipole points. The results of DDA theoretical calculation with the number of even poles greater than 10,000 are accurate and convergent [48]. In this paper, the number of dipole points is at least 13900 , which makes the theoretical study in this paper significant. In addition to the above geometric parameters, there is another important factor: the dielectric function of noble metals. The dielectric function of the $\mathrm{Au}-\mathrm{Ag}$ alloy can be expressed as:

$$
\varepsilon=(1-\beta) \varepsilon_{A u}+\beta \varepsilon_{A g}
$$

Where $\beta$ is the atomic fraction of gold, where $\varepsilon_{A u}$ and $\beta \varepsilon_{A g}$ are the dielectric functions of gold and silver [49].

\section{The Au-Ag alloy nanocages model}

In the theoretical calculation of DDA, we establish Au-Ag alloy nanocages with two metal components of gold and silver evenly distributed, with a square hole in the center of each surface, and a hollow inside. In detail, the hole size (D) and the Au-Ag ratio are adjustable. The twodimensional graphs (x-o-y plane) of the model are illustrated in Fig. 1, and the origin of the coordinates is set at the center of the Au-Ag alloy nanocages. The wall length of the Au-Ag alloy nanocages is fixed at $A=40 \mathrm{~nm}$, the wall thickness is fixed at $\mathrm{h}=5 \mathrm{~nm}$, the value of the hole $\mathrm{D}$ can be increased from 5 to $28 \mathrm{~nm}$, and the value of the Au-Ag ratio can be changed from 1:4 to 1:0. Specifically, as shown in Figs. 1(a)-(b), an adjustable Au-Ag alloy nanocages model is formed by changing the Au composition and hole size. Because the inner hollow cavity is connected to the surrounding dielectric, the environmental dielectric constant inside and outside the $\mathrm{Au}-\mathrm{Ag}$ alloy nanocages are set to be the same during the simulation and calculation of the refractive index sensitivity(denoted as $\varepsilon$ ). In this model, the minimum number of dipole points of the Au-Ag alloy nanocages is 13900 , which can ensure the accuracy and convergence of the DDA calculation results. Besides, the direction of the incident plane wave $\mathrm{K}$ is parallel to the $+\mathrm{x}$ axis, and the direction of the incident electric field $\mathrm{E} 0$ is along the $+\mathrm{y}$ axis. 


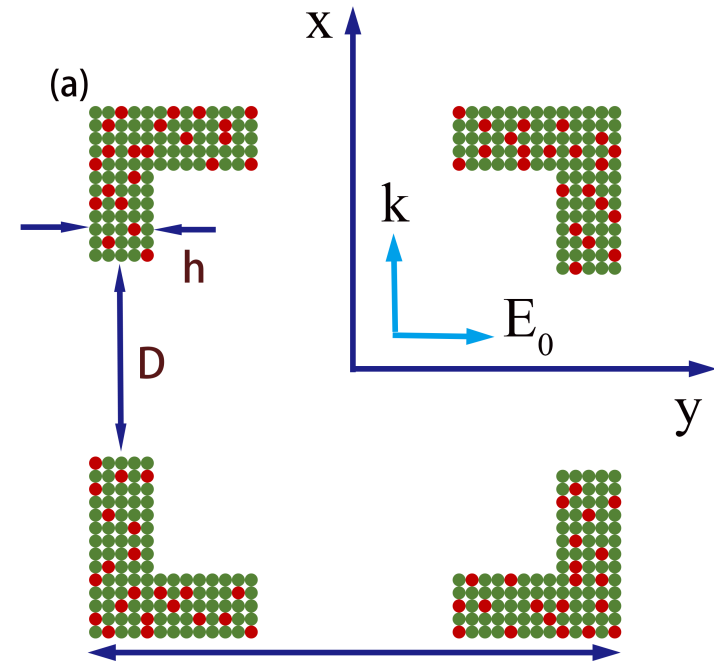

A (b)
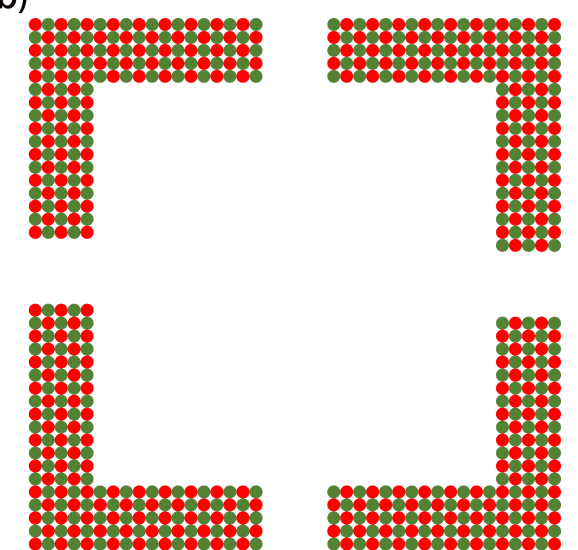

Ag atom

Fig. 1 The two-dimensional x-o-y plane model of Au-Ag alloy nanocages. The wall thickness $\mathrm{h}$ and the wall length A are fixed at $5 \mathrm{~nm}$ and $40 \mathrm{~nm}$, respectively. The $\mathrm{Au}-\mathrm{Ag}$ ratio and hole size D are variable. (a) High $\mathrm{Ag}$ composition, large hole size. (b) There is as much Au composition as Ag composition, small hole size

\section{Results and discussion}

\section{Optical properties of the Au-Ag alloy nanocages}

\section{Extinction spectra of Au-Ag alloy nanocages with different geometric parameters}

To determine the effect of the Au-Ag ratio and the hole size on the refractive index sensitivity factor of the Au-Ag alloy nanocages, the extinction spectra of different $\mathrm{Au}-\mathrm{Ag}$ ratios and different hole sizes in two environmental dielectric constants have been compared in Fig. 2. In the theoretical calculation of DDA, The wall length A is $40 \mathrm{~nm}$ and the wall thickness is $5 \mathrm{~nm}$. We observe one LSPR peak from the corresponding spectrum. It can be found that LSPR is sensitive to changes in parameters such as the Au-Ag ratio, the hole size and the environmental dielectric constant. To analyze conveniently, the main parameters of the Au-Ag alloy nanocages are denoted as A-h-D. In detail, when the environmental dielectric constant changes from 1.00 to 1.33 , the redshift of LSPR peak is $115 \mathrm{~nm}$ when the $\mathrm{Au}-\mathrm{Ag}$ ratio is 1:4 and $106 \mathrm{~nm}$ when the $\mathrm{Au}-\mathrm{Ag}$ ratio is 4: 1 . At the same time, it is found that the redshift of the LSPR peak with the $\mathrm{D}=10 \mathrm{~nm}$ is $141 \mathrm{~nm}$, while that of the 
LSPR peak with the $\mathrm{D}=5 \mathrm{~nm}$ is only $115 \mathrm{~nm}$ under the same conditions. From this comparison, decreasing the Au-Ag ratio and increasing the hole size can improve the refractive index sensitivity of the Au-Ag alloy nanocages, but the LSPR peak redshift with large hole size is much larger than that with low $\mathrm{Au}-\mathrm{Ag}$ ratio, which indicates that hole size can better control the refractive index sensitivity. Therefore, the plasmon optical sensitivity of the Au-Ag alloy nanocages can be improved by optimizing the alloy composition and the hole size. The research groups of Zhu et al. [1], Liaw et al [15]. and Chen et al. [4] also reported similar results, the greater the porosity of gold nanocages, the more significant the redshift of LSPR peak.

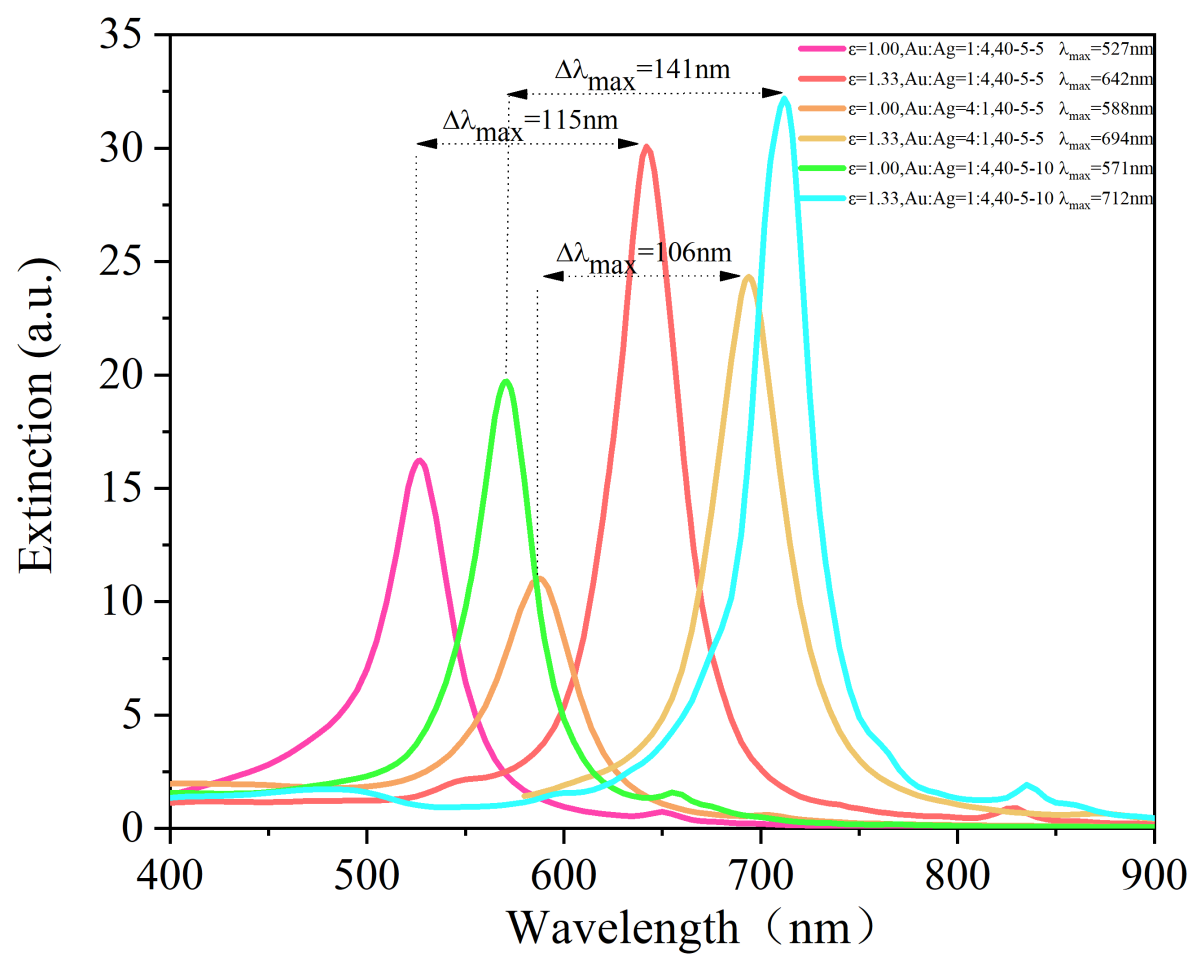

Fig. 2 Extinction spectrum of Au-Ag alloy nanocages with two environmental dielectric constants $(\varepsilon=1.00 、 1.33)$ for different Au-Ag ratios (1:4, 4:1) and different hole sizes ( $\mathrm{h}=5 \mathrm{~nm}, 10 \mathrm{~nm})$

It is well known that gold nanoparticles have good biocompatibility, stability and silver nanoparticles have high sensitivity, while Au-Ag alloy nanoparticles can have the advantages of both gold and silver nanoparticles, which has aroused the interest of many researchers. To further research the effect of the Au-Ag ratio and the hole size on the LSPR spectrum of the Au-Ag alloy nanocages and the effect of the environmental dielectric constant on the plasmon peak wavelength of the Au-Ag alloy nanocages at different Au-Ag ratios and different hole sizes, Fig. 3 has plotted the extinction spectrum with different geometric parameters. Fig. 3(a) and Fig. 3(c) compare the 
LSPR spectrum with small and large hole sizes, respectively, at different Au-Ag ratios. The LSPR peak exhibits approximately linear redshift with the increase of the Au component, and the LSPR peak offset increases and intensity decreases. The change of LSPR peak is due to the decrease of the dielectric constant of alloy material caused by the increase of Au composition, which leads to the increase of LSPR peak wavelength [50] and the decrease of LSPR peak energy in the environmental dielectric constant.

Fig. 3(b) and Fig. 3(d) respectively show the LSPR spectrum with small and large hole sizes at different environmental dielectric constants. When the environmental dielectric constant increases, the LSPR peak also shows approximate linear redshift, which is similar to the result of Lee's research group about the sensitivity of LSPR peak of Au nanocubes and Ag nanocubes to refractive index [16]. Interestingly, the LSPR peak strength of the small hole increases with the increase of the environmental dielectric constant. On the contrary, the LSPR peak strength of large hole decreases with the increase of the environmental dielectric constant. This may be because the hole size affects the local electric field distribution and local electric field strength [1]. Therefore, the effects of the Au-Ag ratio, hole size, and environmental dielectric constant on the LSPR peak can be discussed through the local electric field distribution to clarify the physical mechanism of the effect of geometric parameters on the LSPR peak.

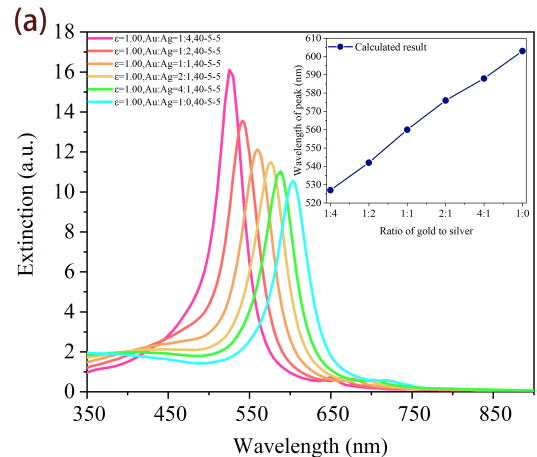

(c)

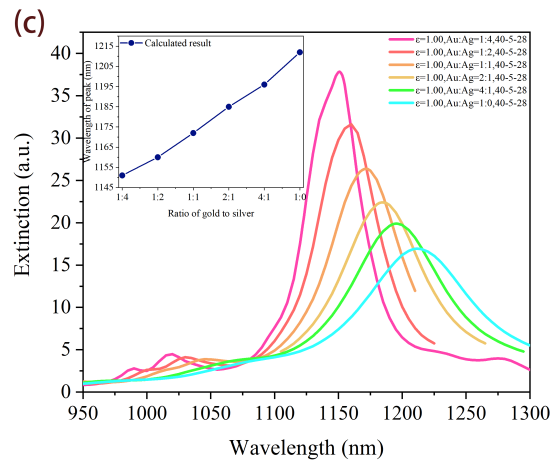

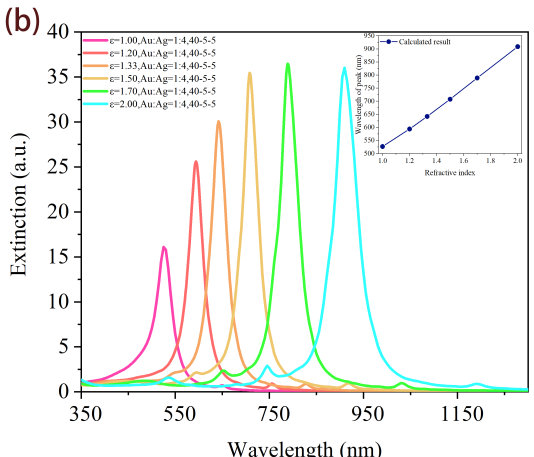

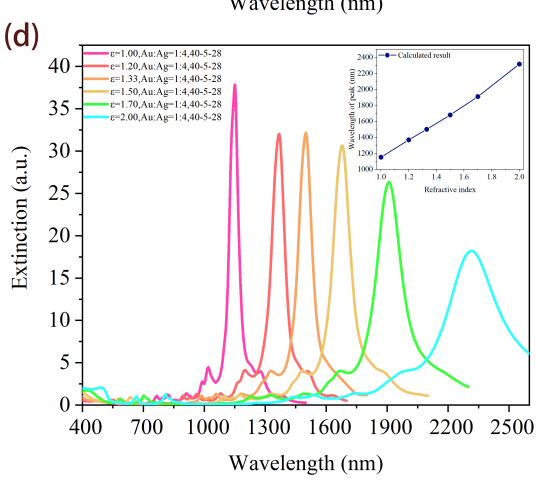

Fig. 3 DDA calculation results of extinction spectrum with different geometric parameters. (a) The Au-Ag ratios are 
1: $0,1: 1,1: 2,1: 4,2: 1$, and 4: 1, respectively, with fixed $\mathrm{h}=5 \mathrm{~nm}$. (b) The environmental dielectric constants $(\varepsilon)$ are 1.00, 1.20, 1.33, 1.50, 1.70, and 2.00, respectively, with fixed $\mathrm{h}=5 \mathrm{~nm}$ and $\mathrm{Au}: \mathrm{Ag}=1: 4$. (c) The $\mathrm{Au}-\mathrm{Ag}$ ratios are 1: $0,1: 1,1: 2,1: 4,2: 1$, and 4: 1, respectively, with fixed $\mathrm{h}=28 \mathrm{~nm}$. (d) The environmental dielectric constants ( $\varepsilon$ ) are $1.00,1.20,1.33,1.50,1.70$, and 2.00, respectively, with fixed $\mathrm{h}=28 \mathrm{~nm}$ and $\mathrm{Au}: \mathrm{Ag}=1: 4$. The inset shows the LSPR peak as a function of corresponding parameters.

\section{Refractive index sensitivity of Au-Ag alloy nanocages with different Au components}
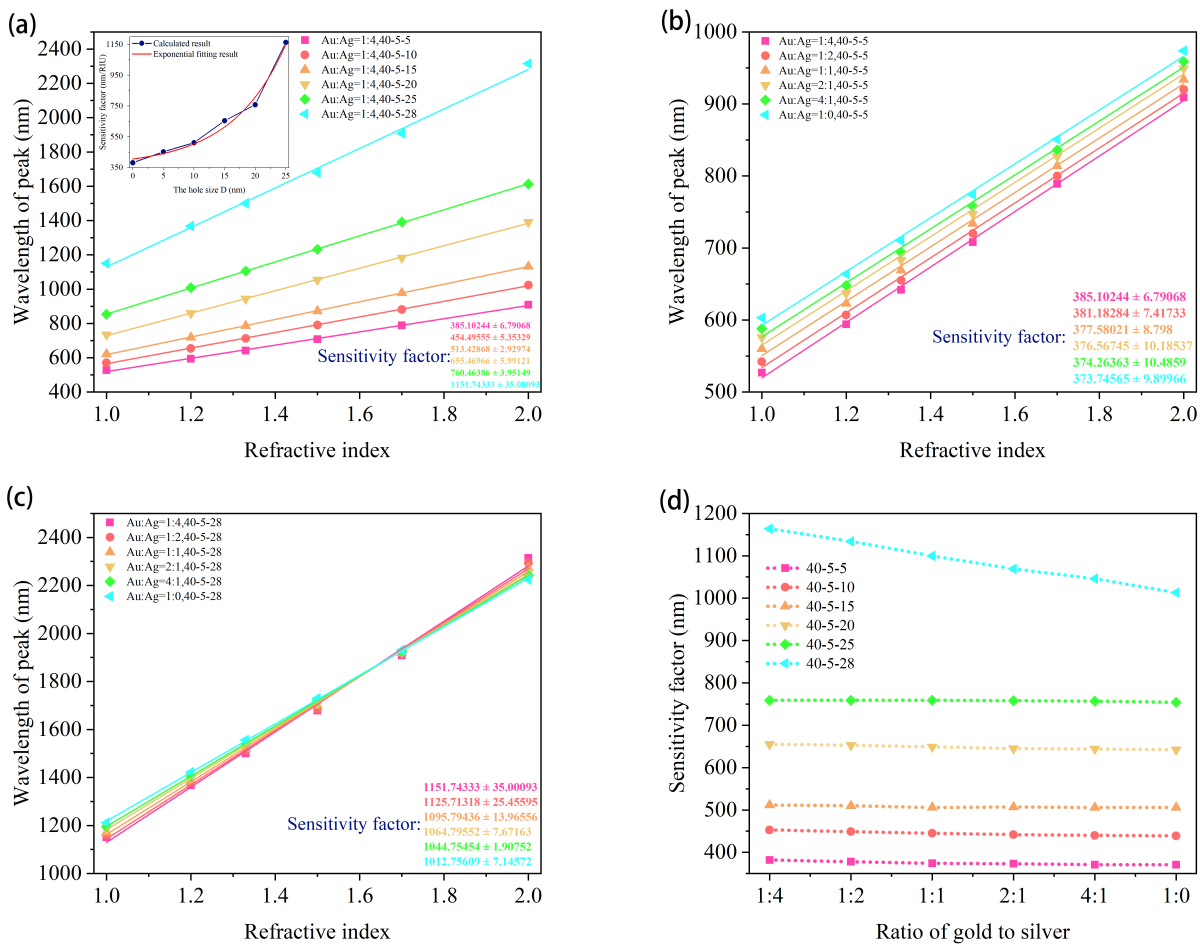

Fig. 4 (a) The LSPR peak versus the environmental dielectric constant for different hole sizes, The inset shows the relationship between the refractive index sensitivity and hole size. (b) The LSPR peak with $\mathrm{D}=5 \mathrm{~nm}$ versus the environmental dielectric constant for different $\mathrm{Au}-\mathrm{Ag}$ ratios. (c) The LSPR peak with $\mathrm{D}=28 \mathrm{~nm}$ versus the environmental dielectric constant for different $\mathrm{Au}-\mathrm{Ag}$ ratios. (d) The dependence of the refractive index sensitivity on the Au-Ag ratio with different hole sizes

To determine the characteristic of the LSPR peak, Fig. 4(a) depicts the LSPR peak as a function of the environmental dielectric constant of different hole sizes. One can found that the LSPR peak increases approximately linearly with the increase of the environmental dielectric constant. And the slope of the curve increases approximately exponentially with the increase of the hole size. For instance, When the hole is $\mathrm{h}=5 \mathrm{~nm}$, the LSPR peak increases to $890 \mathrm{~nm}$ as the environmental 
dielectric constant increases from 1.00 to 2.00 . When we systematically increase the hole size from 5 to $28 \mathrm{~nm}$, the LSPR can reach $2315 \mathrm{~nm}$. Similar results have been found in the studies of Zhu et al. [10], Tam et al. [51], and Prodan et al. [52], it was found that the LSPR peak redshifted with the increase of the environmental dielectric constant, which were attributed to the fact that the increase in the environmental dielectric constant decreased the restoring force of the oscillating electrons of the metal nanoparticles, resulting in the reduction in LSPR peak energy.

As we all know, the refractive index sensitivity factor is defined as the change in the LSPR peak position per unit refractive index (RIU), denoted as $\Delta \lambda_{\max } / \Delta \mathrm{n}$. In this paper, the refractive index sensing characteristics with different $\mathrm{Au}-\mathrm{Ag}$ ratios and different hole sizes are investigated. In our theoretical calculation, the refractive index sensitivity is determined by calculating the extinction spectrum under different parameters with the environmental dielectric constant varying from 1.0 to 2.0. As shown in the simulation results of Figs. 4(a)-(c), according to the definition of the refractive index sensitivity factor, it can be seen that the slope of the fitting curve can be expressed as the refractive sensitivity factor.

The curves in Figs. 4(b)-(c) show the relationship between the LSPR peak and the environmental dielectric constant at different $\mathrm{Au}-\mathrm{Ag}$ ratios. For the small hole size $\mathrm{D}=5 \mathrm{~nm}$ and the $\mathrm{Au}-\mathrm{Ag}$ ratio of 1: 4, when the environmental dielectric constant increases from 1.0 to 2.0, the LSPR peak redshifts to $909 \mathrm{~nm}$. When the $\mathrm{Au}-\mathrm{Ag}$ ratio increases to 1: 0, the LSPR peak can reach $974 \mathrm{~nm}$. However, for large hole size $\mathrm{D}=28 \mathrm{~nm}$ and $\mathrm{Au}-\mathrm{Ag}$ ratio of 1: 4, the LSPR peak increases from 1151 $\mathrm{nm}$ to $2315 \mathrm{~nm}$ at the same refractive index change. When the $\mathrm{Au}-\mathrm{Ag}$ ratio increases to $1: 0$, the LSPR peak decreased slightly to $2225 \mathrm{~nm}$. Overall, the LSPR peak redshift with the increase of the environmental dielectric constant. More interestingly, the LSPR peak in the small hole size moves to the longer wavelength with the increase of the Au component. However, the opposite result is observed in the large hole size and the environmental dielectric constant of 2.0, where the LSPR shifts to the shorter wavelength with the increase of the Au composition.

To more clearly observe the relationship between the refractive index sensitivity and the AuAg ratio, Fig. 4(d) shows the refractive index sensitivity with different geometric parameters. The results show that the Au-Ag ratio has different effects on the refractive index sensitivity with different hole sizes. Specifically, when the hole size is increased from 5 to $25 \mathrm{~nm}$, the Au-Ag ratio 
has little effect on the refractive index sensitivity. On the contrary, when the hole size is $28 \mathrm{~nm}$, the refractive index sensitivity decreases from $1164 \mathrm{~nm} / \mathrm{RIU}$ to $1013 \mathrm{~nm} / \mathrm{RIU}$ with the increase of the Au composition, that is, when the hole size is large enough, the Au-Ag ratio can effectively control the refractive index sensitivity. The refractive index sensitivity of Au-Ag alloy nanocages is higher than that of gold nanocages studied by Zhu et al. [1]. Therefore, the Au-Ag alloy nanocages with large hole size and low Au composition have excellent refractive index sensitivity. This means that Au-Ag alloy nanocages may be used to make more sensitive optical sensors.

\section{Two-dimensional local electric field distribution of Au-Ag alloy nanocages Analysis}

\section{The physical mechanism of component-controlled LSPR}

The results of Figs. 3(a)-(b) show that the Au-Ag ratio plays an important role in affecting LSPR peak properties. To clarify the physical mechanism of the effect of the Au-Ag ratio on the LSPR peak, the local electric field distributions of y-o-z sections with different Au-Ag ratios at the resonance wavelengths corresponding to different geometric parameters are calculated. The DDA simulation results are shown in Fig. 5 and Fig. 7. Notably, the local electric field strength of the LSPR peak calculated by DDA decreases sharply with the distance increase from the surface, resulting in a large error in the theoretical calculation [1]. Therefore, the local field distance on the surface of Au-Ag alloy nanocages calculated in this paper is $0.25 \mathrm{~nm}$, and the dipole distance is 1.0 $\mathrm{nm}$, which guarantees the accuracy and reliability of DDA theoretical calculation.

Fig. 5 shows the local electric field distributions for different $\mathrm{Au}-\mathrm{Ag}$ ratios at $\mathrm{D}=5 \mathrm{~nm}$. We find that the strong electric field is concentrated on the outer tip and the hole surface, but the local electric field at the outer tip is stronger. This is due to the electric field focusing effect of the tip $[1,9]$. On the contrary, small hole surface area and large $\{100\}$ crystal plane disperse polarized charges, so plasmon coupling between hole surfaces is relatively weak. However, it should be noted that the $\mathrm{Au}-\mathrm{Ag}$ ratio does not affect the electric field direction on the cavity surface. In detail, when the AuAg ratio is 1:4, as shown in Fig. 5(a1) and Fig. 5(a2), the outer surface and the hole surface generate the $+y$ direction electric field in the same direction as the incident polarization field. The electric field generated by the cavity is in the -y direction. Interestingly, when the $\mathrm{Au}-\mathrm{Ag}$ ratio changes from 1: 4 to 1: 0, compared with Fig. 5(a1) and Fig. 5(a2), the electric field directions in Figs. 5(b1)-(d1) 
and Figs. 5(b2)-(d2) remains unchanged, and the local enhancement factor decreases with the increase of the Au composition. This may be due to the reduction of the dielectric constant of the $\mathrm{Au}-\mathrm{Ag}$ alloy material reducing the polarized charges density of the outer tip and the hole surface, thereby weakening the electric field focusing effect of the outer tip and plasmon coupling between the two hole surfaces. In addition, a small amount of positive (negative) charges distribution is observed on the left side (right side) of the cavity in Fig. 5(d2), which meant that the effect of the polarized electric field in the environmental dielectric constant on the electric field distribution in the cavity is increased.
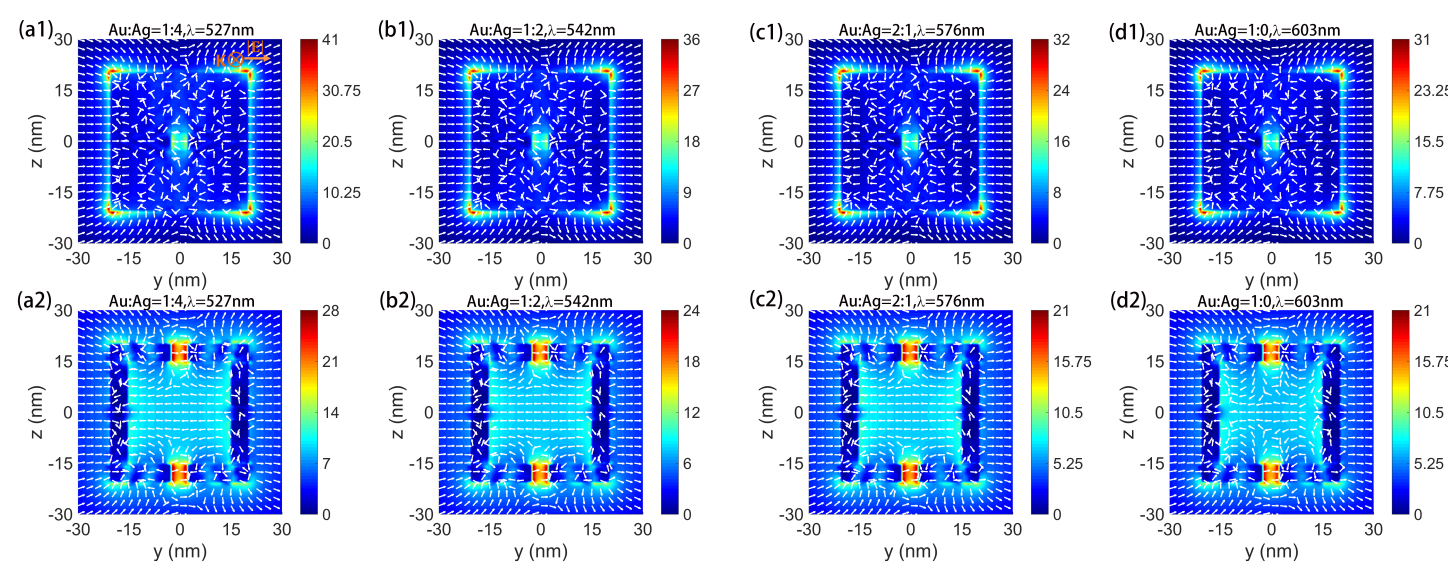

Fig. 5 Local field vector distribution in the y-o-z plane of Au-Ag alloy nanocages. Geometric parameters include the environmental dielectric constant 1.00, local electric field enhancement factor $|\mathrm{E} / \mathrm{E} 0|$, wall thickness h fixed at 5 $\mathrm{nm}$, and adjustable the Au-Ag ratio(1:4,1:2,2:1,1:0). (a1)-(d1) The y-o-z middle plane (x=0). (a2)-(d2) The y-o-z surface plane $(x=20)$.

The appearance and movement of noble metal nanoparticles LSPR peak are closely related to the Coulomb restoring force between the positive and negative charges. The change of Coulomb restoring force leads to the change of LSPR peak resonance energy. It is well known that the electron relaxation time of gold is shorter than that of silver [53], which means that the electron scattering of gold is stronger than that of silver. Therefore, when the Au component is large, the LSPR peak energy decreases and the plasmon resonance weakens, and the LSPR peak undergoes redshift and LSPR peak intensity decreases, as shown in Fig. 3(a) and Fig. 3(c). 

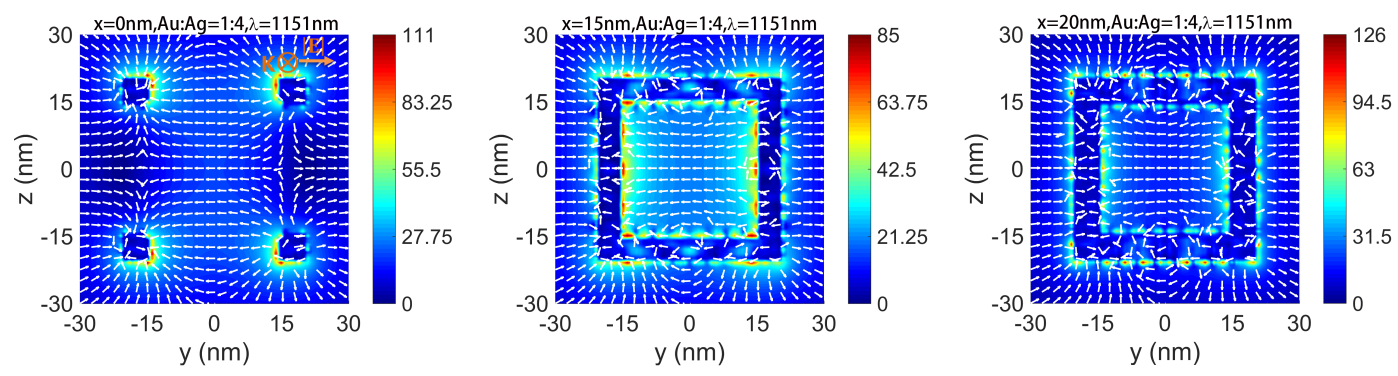

Fig. 6 Local electric field distributions at different cross-sections in the y-o-z plane of Au-Ag alloy nanocages with $\varepsilon=1.00, \mathrm{~h}=28 \mathrm{~nm}$ and the $\mathrm{Au}-\mathrm{Ag}$ ratio of $1: 4$

To elucidate the "hot spots" distribution for large hole size, Fig. 6 shows the local electric field distribution of different y-o-z sections of large hole size. Fig. 6 shows a completely different result from Fig. 5, the local electric field is uniformly distributed on the whole surface, and the strong local electric field is concentrated on the hole surface and the inner corner of the Au-Ag alloy nanocages. This may be due to the increase in the effective coupling area of the large hole size, resulting in more polarized charges concentration on the hole surface and less polarized charges at the outer tip. Besides, the local electric field is uniformly distributed due to the plasmon coupling excited by the shorter distance between the outer wall and the inner wall. More polarized charges on the hole surface and the electric field focusing effect at the inner corner indicate that there is a stronger electric field at the inner corner. Therefore, the hole size affects the local electric field distribution.
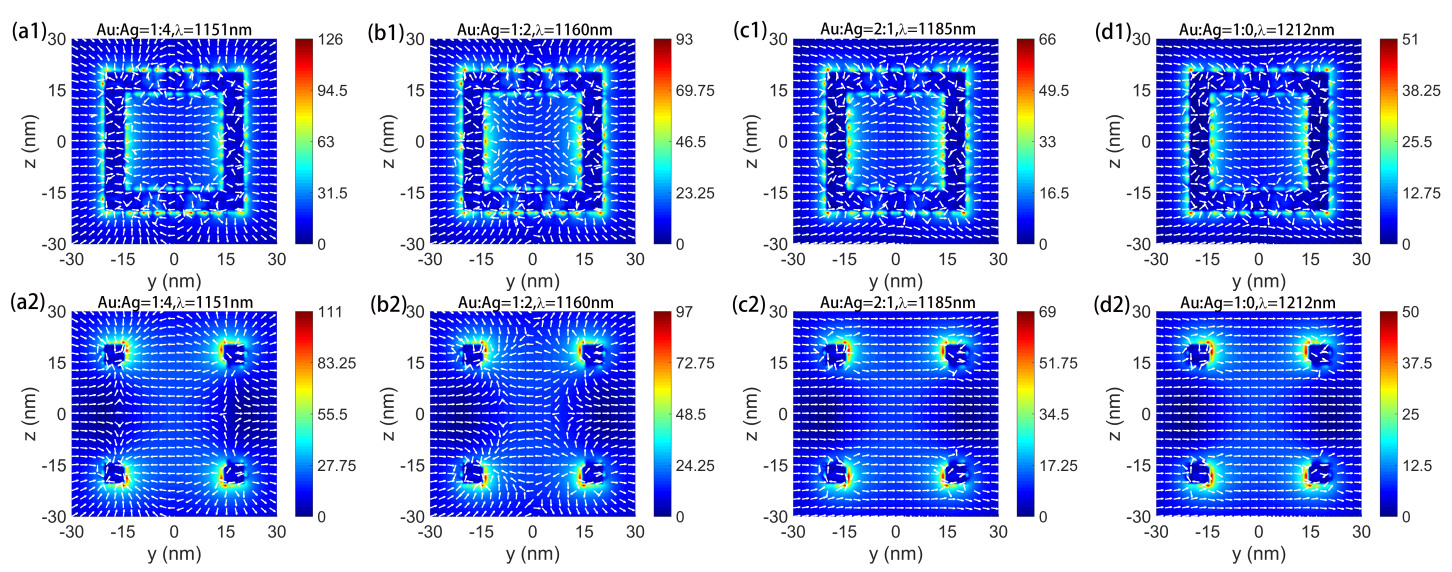

Fig. 7 Local field vector distribution in the y-o-z plane of Au-Ag alloy nanocages. Geometric parameters include the environmental dielectric constant 1.00, local electric field enhancement factor |E/E0|, wall thickness h fixed at 28 $\mathrm{nm}$, and adjustable the Au-Ag ratio(1:4,1:2,2:1,1:0). (a1)-(d1) The y-o-z middle plane (x=0), (a2)-(d2) The y-o-z 
surface plane $(\mathrm{x}=20)$.

Fig. 7 shows the local electric field distribution of the LSPR peak, in which $\mathrm{D}=28 \mathrm{~nm}$ and AuAg ratio change. Compared with Fig. 5, the electric field distribution on the cavity and hole surfaces with large hole size can be changed by the Au-Ag ratio. The larger hole size increases the environmental dielectric composition on the hole surface, which makes the hole surface possibly affected by more polarized electric fields in the environmental dielectric constant. At the same time, more environmental dielectric composition is distributed on the cavity surface. Finally, the incident polarized field, the polarized field on the outer surface and the polarized field in the environmental dielectric constant are superimposed to form the electric fields on the cavity and hole surface. Therefore, the electric field distribution of the cavity and hole surface may change. It should be noted that the electric field on the hole surface may affect the electric field distribution of the cavity.

In detail, Fig. 7(a1) and Fig. 7(a2) show that the outer surface electric field is along the $+y$ direction, and the electric fields of hole and cavity are along the -y direction. When the $\mathrm{Au}-\mathrm{Ag}$ ratio increases to 1:2, a small amount of positive (negative) charges are distributed on the left (right) side of the hole and cavity surface. The possible reasons are that the increase of environmental dielectric composition on the surface of hole and cavity makes the polarized field in the environmental dielectric constant have greater influence, and the increase of gold composition makes the plasmon resonance on the hole surface weaken. Therefore, the electric fields in the -y direction of hole and cavity weaken, while the electric field in the $+y$ direction of the outer surface increases. Compared with Fig. 7(a1) and Fig. 7(a2), the electric fields of hole and cavity in Figs. 7(c1)-(d1) and Figs. 7(c2)-(d2) are along the $+y$ direction when the Au-Ag ratio is 2: 1 and 1: 0 , which means that more and more positive (negative) charges are distributed on the left (right) side of hole and cavity, and the $+y$ direction electric field enhanced by the outer surface affects the electric field distribution of the hole and cavity. With the increase of Au component, the electric field in $+y$ direction on the outer surface increases, and the electric fields in $-\mathrm{y}$ direction of hole and cavity decrease, so the local electric field enhancement factor decreases.

The physical mechanism of the hole size-dependent refractive index sensing of nanocages by tuning the alloy composition 

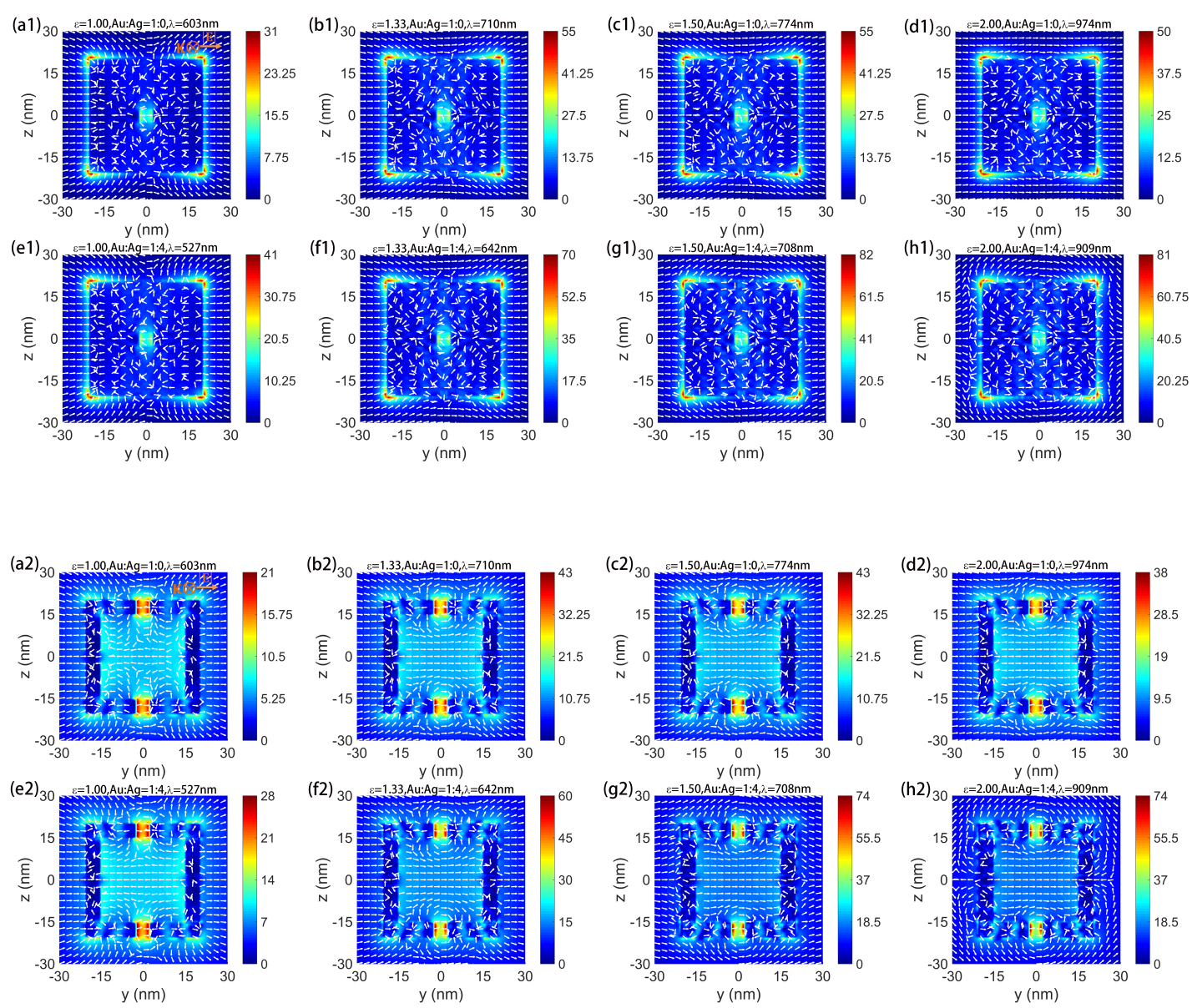

Fig. 8 Local electric field factor defined as $|\mathrm{E} / \mathrm{E} 0|$ and field vector distributions on different y-o-z planes for $\mathrm{Au}-\mathrm{Ag}$ alloy nanocages with $\mathrm{D}=5 \mathrm{~nm}$. (a1)-(d1) The y-o-z surface plane ( $\mathrm{x}=20)$. (a2)-(h2) The $\mathrm{y}-\mathrm{o}-\mathrm{z}$ middle plane ( $\mathrm{x}=0$ ).
(a1),
(a2) $\varepsilon=1.00, \mathrm{Au}: \mathrm{Ag}=1: 0, \lambda=603$
$\mathrm{nm}$;
(b1),
(b2) $\varepsilon=1.33, \mathrm{Au}: \mathrm{Ag}=1: 0, \lambda=710 \quad \mathrm{~nm}$;
(c1),
(c2)

$\varepsilon=1.50, \mathrm{Au}: \mathrm{Ag}=1: 0, \lambda=774 \mathrm{~nm} ;(\mathrm{d} 1),(\mathrm{d} 2) \varepsilon=2.00, \mathrm{Au}: \mathrm{Ag}=1: 0, \lambda=974 \mathrm{~nm} ;(\mathrm{e} 1),(\mathrm{e} 2) \varepsilon=1.00, \mathrm{Au}: \mathrm{Ag}=1: 4, \lambda=527 \mathrm{~nm} ;$ (f1), (f2) $\varepsilon=1.33, \mathrm{Au}: \mathrm{Ag}=1: 4, \lambda=642 \mathrm{~nm} ;(\mathrm{g} 1),(\mathrm{g} 2) \varepsilon=1.50, \mathrm{Au}: \mathrm{Ag}=1: 4, \lambda=708 \mathrm{~nm}$; (h1), (h2) $\varepsilon=2.00, \mathrm{Au}: \mathrm{Ag}=1: 4$, $\lambda=909 \mathrm{~nm}$.

The results of Figs. 4(b)-(c) show that the increase of the Ag component in the large hole size can improve the refractive index sensitivity. To investigate the influence of $\mathrm{Au}-\mathrm{Ag}$ ratio on refractive index sensitivity, the field vectors of different refractive indices with different $\mathrm{Au}-\mathrm{Ag}$ ratios and different hole sizes are plotted in Fig. 8 and Fig. 9. The refractive indices are 1.00, 1.33, 1.50 and 2.00 , and $\mathrm{A}$ and $\mathrm{h}$ are fixed at $40 \mathrm{~nm}$ and $5 \mathrm{~nm}$, respectively. When $\mathrm{D}=5 \mathrm{~nm}$, the refractive index changes the cavity electric field direction, while when $\mathrm{D}=28 \mathrm{~nm}$ and the Au-Ag ratio is 1:4, the direction of the hole and cavity electric field change inversely. This may be because more 
environmental dielectric constants are distributed on the surfaces of cavity and large hole, and the polarized electric field in the environmental dielectric constant has a greater influence on the electric fields on the surfaces of cavity and large hole size. In addition, the hole size can affect the distribution of "hot spots" and the electric field distribution on the hole surface.

The local electric field distribution of the small hole size is shown in Fig. 8. When the refractive index is 1.00 , the electric fields of the outer and hole surfaces are in the +y direction, and the cavity electric field is in the $-\mathrm{y}$ direction. When the refractive index increases $(\varepsilon=1.33,1.50)$, the cavity electric field is in the $+y$ direction and the local electric field enhancement factor increases. The increased environmental dielectric constant enhances the polarized electric field in the environmental dielectric constant, and the polarized electric field reduces the restoring force of oscillating electrons on the outer surface and the hole surface, resulting in the decrease of LSPR peak energy and the redshift of LSPR peak [10]. At the same time, the increased environmental dielectric constant increases the electron dipole moment on the outer and hole surface [54], and further leads to the enhancement of plasmon coupling on the outer and hole surface, and thus the local electric field enhancement factor becomes larger. It should be noted that the enhanced $+y$ electric field weakens the -y electric field on the cavity surface, and the $+y$ electric field with sufficient intensity may cause a change in the electric field direction of the cavity. Further, a small amount of positive (negative) charges on the left (right) side of the outer surface is found in Fig. 8 (g1) and Fig. $8(\mathrm{~g} 2)$. When the refractive index is 2.00 , the local electric field enhancement factor decreases slightly. The outer surface, which distributes more polarized charges, reduces the polarized charges density on the hole surface so that plasmon resonance on the hole surface is relatively weak. Of interest, the local electric field enhancement factors in Fig. 8(h1) and Fig. 8 (h2) decrease only slightly because there are more positive charges on the left side of the outer surface, resulting in a stronger -y electric field.

Fig. 9 shows the local electric fields with different refractive indices for large hole size. When the $\mathrm{Au}-\mathrm{Ag}$ ratio is 1:0, with the increase of the refractive index, the enhanced polarized field in the environmental dielectric constant weakens the $+y$ direction electric field on the hole surface [1], and thus the weakening of the local electric field enhancement factor is observed. When the Au-Ag ratio is $1: 4$, the refractive index increases from 1.00 to 1.33 , and the electric fields of the hole and cavity 
surface are along the $-\mathrm{y}$ direction. The $+\mathrm{y}$ electric field on the outer surface is enhanced by the increase of the dipole moment on the outer surface. The enhanced +y electric field may affect the electric field distributions on the hole and cavity surfaces, so when the refractive index increases to 1.50 and 2.00 , the electric fields on the hole and cavity surface are along the $+y$ direction. With the increase of the refractive index, the $+y$ direction electric field increases, and then the local electric field enhancement factor decreases. It is noteworthy that the large hole size distributes more environmental dielectric components, and more Ag components can enhance the plasmon resonance, and the $+\mathrm{y}$ direction electric field of the Au-Ag alloy nanocages is enhanced due to the increase of the refractive index. Therefore, the Au-Ag alloy nanocages with large hole size and more $\mathrm{Ag}$ components have higher refractive index sensitivity.
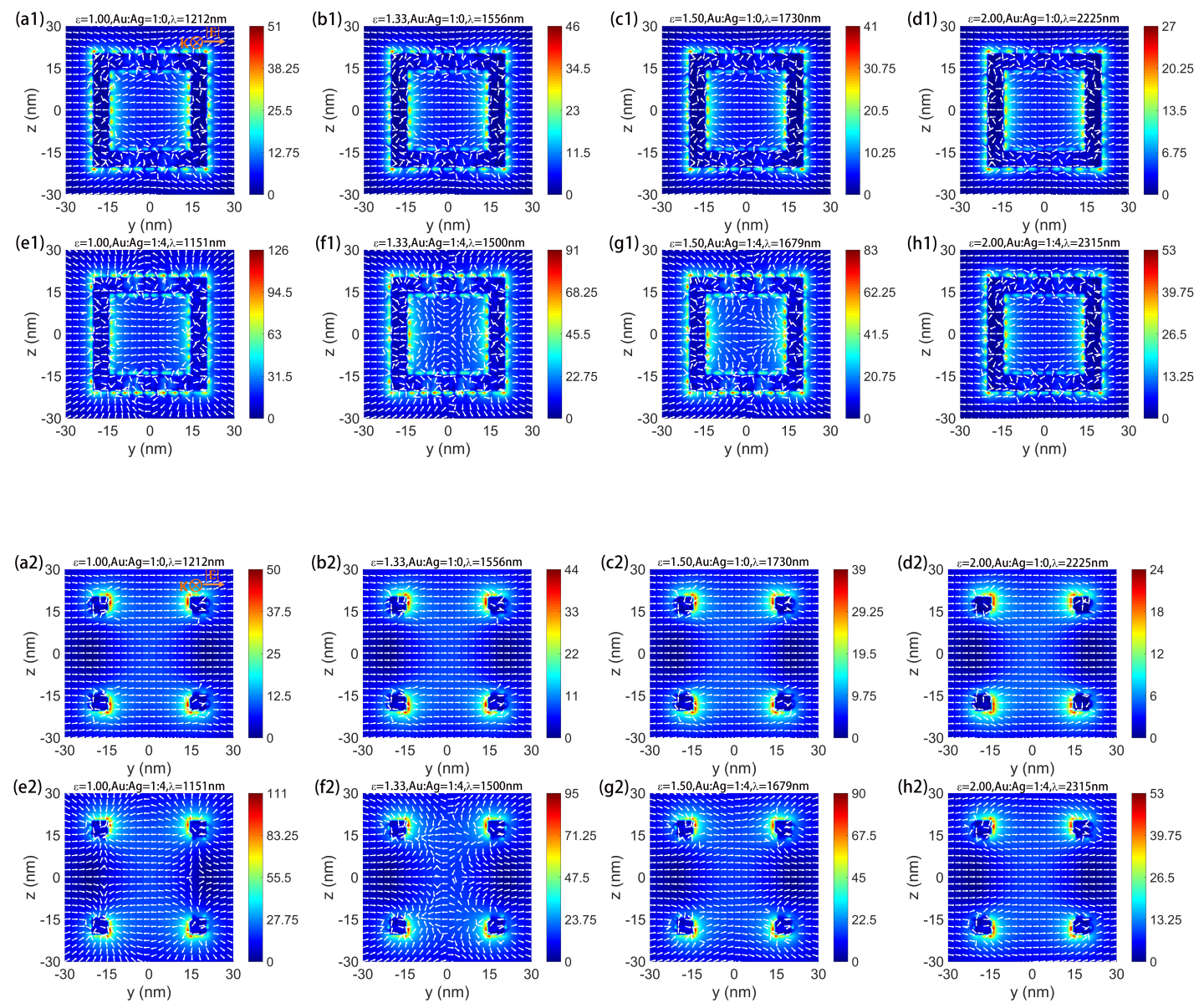

Fig. 9 Local electric field factor defined as $|\mathrm{E} / \mathrm{E} 0|$ and field vector distributions on different y-o-z planes for Au-Ag alloy nanocages with $\mathrm{D}=28 \mathrm{~nm}$. (a1)-(d1) The $\mathrm{y}-\mathrm{o}-\mathrm{z}$ surface plane ( $\mathrm{x}=20)$. (a2)-(h2) The $\mathrm{y}-\mathrm{o}-\mathrm{z}$ middle plane ( $\mathrm{x}=0$ ).
(a1),
(a2) $\varepsilon=1.00, \mathrm{Au}: \mathrm{Ag}=1: 0, \lambda=1212$
$\mathrm{nm}$.
(b1),
(b2) $\varepsilon=1.33, \mathrm{Au}: \mathrm{Ag}=1: 0, \lambda=1556$
nm. (c1) 
(fl)

(f2) $\varepsilon=1.33, \mathrm{Au}: \mathrm{Ag}=1: 4, \lambda=1500 \quad \mathrm{~nm}$

(g1),

(g2) $\varepsilon=1.50, \mathrm{Au}: \mathrm{Ag}=1: 4, \lambda=1679$

$\mathrm{nm}$.

(h1),

$\varepsilon=2.00, \mathrm{Au}: \mathrm{Ag}=1: 4, \lambda=2315 \mathrm{~nm}$.

In general, the increase of Au composition and refractive index cause the LSPR peak redshift of the nanoparticles [53, 55]. Interestingly, when A-h-D is $40-5-28 \mathrm{~nm}$ and the refractive index $\varepsilon$ is 2.00 , the LSPR of the alloy nanocages blueshift with the increase of Au composition. The possible reason is that the stronger additional +y direction electric field is formed on the hole surface with low $\mathrm{Au}$ composition ( $\mathrm{Au}: \mathrm{Ag}=1: 4)$, Therefore, the restoring force of the $\mathrm{Au}-\mathrm{Ag}$ alloy nanocages LSPR peak is lower and the LSPR peak wavelength is longer.

\section{Conclusions}

In conclusion, the plasmon extinction spectrum of $\mathrm{Au}-\mathrm{Ag}$ alloy nanocages with different $\mathrm{Au}-$ $\mathrm{Ag}$ ratios and different hole sizes and the refractive index sensitivity of $\mathrm{Au}-\mathrm{Ag}$ alloy nanocages are theoretically studied by DDA. The calculation results based on DDA theory show that the LSPR peak can be adjusted by the alloy composition ratio and hole size. At the same time, it is also found that the refractive index sensitivity can be effectively controlled by the alloy composition ratio at the large hole size. The sensitivity decreases linearly with the increase of Au composition but increases exponentially with the increase of hole size. In addition, the local field vector distributions with different hole sizes, different Au-Ag ratios, and different refractive indexes are investigated, which indicates that the field vectors of the hole and cavity are formed by the superposition of the incident field, the electric field formed by the outer surface of the nanocages, and the polarized field formed by the environmental dielectric constant, so the electric field direction of the hole and cavity may be reversely deflected. Moreover, in the case of large hole size, the electric field distributions on the surfaces of the cavity and hole are controlled by the $\mathrm{Au}-\mathrm{Ag}$ ratio and refractive index. Therefore, the refractive index sensitivity can be improved by increasing the $\mathrm{Ag}$ component under the condition of larger hole size, and the Au-Ag alloy nanocages will have the potential to become optical sensors with high refractive index sensitivity. 


\section{Statement}

Funding Information : This work was supported by the National Natural Science Foundation of China under Grant Nos. 11774283 and 61675162 .We also would like to thank the Zhejiang Province Basic Public Welfare Research Project (LGF20H1 80017) for their support in this research.

Conflicts of interest : The authors declare that they have no known competing financial interests or personal relationships that could have appeared to influence the work reported in this paper.

Availability of data and material: the data that support the findings of this study are available from the corresponding author upon reasonable request.

Code availability : Custom code data supporting the results of this study are available from the corresponding author upon reasonable request. The software used is DDSCAT.7.3.

Authors' contributions : Qiu-Xiang Qin: Methodology, Investigation, Data curation, Writing - original draft. Jian-Jun Li: Formal analysis, Writing review \& editing. Jian Zhu: Conceptualization, Resources, Writing review \& editing, Supervision, Funding acquisition. Guo-Jun Weng: Resources, Project administration, Supervision. Jun-Wu Zhao: Conceptualization, Resources, Writing - review \& editing, Supervision.

Ethics approval: This is an observational study. The XYZ Research Ethics Committee has confirmed that no ethical approval is required.

Consent to participate: I would like to declare on behalf of my co-authors that the work described was original research that has not been published previously, and not under consideration for publication elsewhere, in whole or in part. All the authors listed have approved the manuscript that is enclosed.

Consent for publication: No conflict of interest exits in the submission of this manuscript, and manuscript is approved by all authors for publication 


\section{References}

1. Zhu J, Chen JK, Li JJ and Zhao JW (2019) Local dielectric environment-dependent plasmonic optical sensitivity of gold nanocage: from nanobox to nanoframe. Applied Physics A 125:62

2. Rycenga M, Cobley CM, Zeng J, Li W, Moran CH, Zhang Q, Qin D and Xia Y (2011) Controlling the Synthesis and Assembly of Silver Nanostructures for Plasmonic Applications. Chemical Reviews 111:3669-3712

3. Wang Y, Zhang P, Mao X, Fu W and Liu C (2016) Seed-mediated growth of bimetallic nanoparticles as an effective strategy for sensitive detection of vitamin C. Sensors and Actuators B: Chemical 231:95-101

4. Chen JK, Zhao SM, Zhu J, Li JJ and Zhao JW (2020) Colorimetric determination and recycling of $\mathrm{Hg}^{2+}$ based on etching-induced morphology transformation from hollow $\mathrm{AuAg}$ nanocages to nanoboxes. Journal of Alloys and Compounds 828:154392

5. Wang Y, Zhang P, Fu W and Zhao Y (2018) Morphological control of nanoprobe for colorimetric antioxidant detection. Biosens Bioelectron 122:183-188

6. Bao S, Huang S, Liu Y, Hu Y, Wang W, Ji M, Li H, Zhang NX, Song C and Duan S (2017) Gold nanocages with dual modality for image-guided therapeutics. Nanoscale 9:7284-7296

7. Li K, Wang Y, Cai F, Yu J, Wang S, Zhu Z, Chu L, Zhang H, Qian J and He S (2015) Nonlinear optical properties of $\mathrm{Au} / \mathrm{Ag}$ alloyed nanoboxes and their applications in both in vitro and in vivo bioimaging under long-wavelength femtosecond laser excitation. RSC Advances 5:2851-2856

8. Pang B, Yang X and Xia Y (2016) Putting gold nanocages to work for optical imaging, controlled release and cancer theranostics. Nanomedicine (Lond) 11:1715-1728

9. Zhang CH, Zhu J, Li JJ and Zhao JW (2015) Misalign-dependent double plasmon modes "switch" of gold triangular nanoplate dimers. Journal of Applied Physics 117:063102

10. Zhu J and Deng XC (2011) Improve the refractive index sensitivity of gold nanotube by reducing the restoring force of localized surface plasmon resonance. Sensors and Actuators B: Chemical 155:843-847

11. Zhu J, Li JJ and Zhao JW (2013) Improve the refractive index sensitivity of coaxial-cable 
type gold nanostructure: the effect of dielectric polarization from the separate layer. Journal of Nanoparticle Research 15:1721

12. Mayer KM and Hafner JH (2011) Localized Surface Plasmon Resonance Sensors. Chemical Reviews 111:3828-3857

13. Chen H, Kou X, Yang Z, Ni W and Wang J (2008) Shape- and size-dependent refractive index sensitivity of gold nanoparticles. Langmuir 24:5233-5237

14. Sekhon JS, Malik HK and Verma SS (2013) DDA simulations of noble metal and alloy nanocubes for tunable optical properties in biological imaging and sensing. RSC Advances 3:15427-15434

15. Liaw JW, Cheng JC, Ma C and Zhang R (2013) Theoretical Analysis of Plasmon Modes of Au-Ag Nanocages. The Journal of Physical Chemistry C 117:19586-19592

16. Lee YH, Chen H, Xu QH and Wang J (2011) Refractive Index Sensitivities of Noble Metal Nanocrystals: The Effects of Multipolar Plasmon Resonances and the Metal Type. The Journal of Physical Chemistry C 115:7997-8004

17. Edit C, Albert O, Erika V, ádám J, Norbert B, László K, Andrea M and Imre D (2012) Synthesis and characterization of $\mathrm{Ag} / \mathrm{Au}$ alloy and core( $\mathrm{Ag})-\operatorname{shell}(\mathrm{Au})$ nanoparticles. Colloids and Surfaces A: Physicochemical and Engineering Aspects 415:281-287

18. Zhang Q, Cobley CM, Zeng J, Wen LP, Chen J and Xia Y (2010) Dissolving Ag from AuAg Alloy Nanoboxes with $\mathrm{H}_{2} \mathrm{O}_{2}$ : A Method for Both Tailoring the Optical Properties and Measuring the $\mathrm{H}_{2} \mathrm{O}_{2}$ Concentration. The Journal of Physical Chemistry C 114:6396-6400

19. Zhu J, Zhao BZ, Qi Y, Li JJ, Li X and Zhao JW (2018) Colorimetric determination of Hg(II) by combining the etching and aggregation effect of cysteine-modified Au-Ag core-shell nanorods. Sensors and Actuators 255:2927-2935

20. Zhu J, Jia TT, Li JJ, Li X and Zhao JW (2019) Plasmonic spectral determination of Hg(II) based on surface etching of $\mathrm{Au}-\mathrm{Ag}$ core-shell triangular nanoplates: From spectrum peak to dip. Spectrochimica Acta Part A: Molecular and Biomolecular Spectroscopy 207:337347

21. Qi Y, Zhao J, Weng GJ, Li JJ, Li X, Zhu J and Zhao JW (2018) A colorimetric/SERS dualmode sensing for detection of mercury (II) based on rhodanine stabilized gold 
nanobipyramids. Journal of Materials Chemistry C 6:12283-12293

22. Zhu J, Chen XH, Li JJ and Zhao JW (2019) The synthesis of Ag-coated tetrapod gold nanostars and the improvement of surface-enhanced Raman scattering. Spectrochimica Acta Part A: Molecular and Biomolecular Spectroscopy 211:154-165

23. Zhu J, Xu Zj, Weng GJ, Zhao J, Li JJ and Zhao JW (2018) Etching-dependent fluorescence quenching of Ag-dielectric-Au three-layered nanoshells: The effect of inner Ag nanosphere. Spectrochimica Acta Part A: Molecular and Biomolecular Spectroscopy 200:43-50

24. Zhu J, Zhang F, Chen BB, Li JJ and Zhao JW (2015) Tuning the shell thickness-dependent plasmonic absorption of Ag coated Au nanocubes: The effect of synthesis temperature. Materials Science and Engineering: B 199:113-120

25. Chew WS, Pedireddy S, Lee YH, Tjiu WW, Liu YJ, Yang Z and Ling XY (2015) Nanoporous Gold Nanoframes with Minimalistic Architectures: Lower Porosity Generates Stronger Surface-Enhanced Raman Scattering Capabilities. Chemistry of Materials $27: 7827-7834$

26. Mahmoud MA and El-Sayed MA (2009) Aggregation of Gold Nanoframes Reduces, Rather Than Enhances, SERS Efficiency Due to the Trade-Off of the Inter- and Intraparticle Plasmonic Fields. Nano Letters 9:3025-3031

27. McLellan JM, Siekkinen A, Chen JY and Xia YN (2006) Comparison of the surfaceenhanced Raman scattering on sharp and truncated silver nanocubes. Chemical Physics Letters 427:122-126

28. McLellan JM, Li ZY, Siekkinen AR and Xia YN (2007) The SERS Activity of a Supported Ag Nanocube Strongly Depends on Its Orientation Relative to Laser Polarization. Nano Letters 7:1013-1017

29. Zhou F, Li ZY, Liu Y and Xia YN (2008) Quantitative Analysis of Dipole and Quadrupole Excitation in the Surface Plasmon Resonance of Metal Nanoparticles. The Journal of Physical Chemistry C 112:20233-20240

30. Skrabalak SE, Chen JY, Au L, Lu XM, Li XD and Xia YN (2007) Gold Nanocages for Biomedical Applications. Advanced materials (Deerfield Beach, Fla.) 19:3177-3184

31. Wang Y, Wan J, Miron RJ, Zhao Y and Zhang Y (2016) Antibacterial properties and 
mechanisms of gold-silver nanocages. Nanoscale 8:11143-11152

32. Au L, Lu XM and Xia YN (2008) A Comparative Study of Galvanic Replacement Reactions Involving $\mathrm{Ag}$ Nanocubes and $\mathrm{AuCl}_{2}^{-}$or $\mathrm{AuCl}_{4}^{-}$. Advanced materials (Deerfield Beach, Fla.) 20:2517-2522

33. Cao M, Wang M and Ning G (2009) Optimized Surface Plasmon Resonance Sensitivity of Gold Nanoboxes for Sensing Applications. The Journal of Physical Chemistry C 113:12171221

34. Mahmoud MA and El-Sayed MA (2010) Gold Nanoframes: Very High Surface Plasmon Fields and Excellent Near-Infrared Sensors. Journal of the American Chemical Society $132: 12704-12710$

35. Mahmoud MA, Snyder B and El-Sayed MA (2010) Surface plasmon fields and coupling in the hollow gold nanoparticles and surface-enhanced raman spectroscopy. theory and experiment. The Journal of Physical Chemistry C 114:7436-7443

36. Jain PK and El-Sayed MA (2007) Surface plasmon resonance sensitivity of metal nanostructures: physical basis and universal scaling in metal nanoshells. The Journal of Physical Chemistry C 111:17451-17454

37. Mie G (1908) Articles on the optical characteristics of turbid tubes, especially colloidal metal solutions. Annalen der Physik 25:377-445

38. DeVoe H (1964) Optical Properties of Molecular Aggregates. I. Classical Model of Electronic Absorption and Refraction. The Journal of Chemical Physics 41:393-400

39. Purcell EM and Pennypacker CR (1973) Scattering and Absorption of Light by Nonspherical Dielectric Grains. The Astrophysical Journal 186:705-714

40. Draine BT and Flatau PJ (1994) Discrete-Dipole Approximation For Scattering Calculations. Journal of the Optical Society of America A 11:1491-1499

41. Draine BT (1988) The discrete-dipole approximation and its application to interstellar graphite grains. Astrophysical Journal 333:848-872

42. Goodman JJ, Draine BT and Flatau PJ (1991) Application of fast-Fourier-transform techniques to the discrete-dipole approximation. Optics Letters 16:1198-1200

43. Draine BT and Goodman J (1993) Beyond clausius-mossotti - wave propagation on a 
polarizable point lattice and the discrete dipole approximation. Astrophysical Journal 405:685-697

44. Draine BT and Flatau PJ (2008) Discrete-dipole approximation for periodic targets: theory and tests. Journal of the Optical Society of America A 25:2693-2703

45. Flatau PJ and Draine BT (2012) Fast near field calculations in the discrete dipole approximation for regular rectilinear grids. Optics express 20:1247-1252

46. Yurkin MA and Kahnert M (2013) Light scattering by a cube: Accuracy limits of the discrete dipole approximation and the T-matrix method. Journal of Quantitative Spectroscopy and Radiative Transfer 123:176-183

47. Yurkin MA, Maltsev VP and Hoekstra AG (2006) Convergence of the discrete dipole approximation. I. Theoretical analysis. Journal of the Optical Society of America A $23: 2578-2591$

48. Sosa IO, Noguez C and Barrera RG (2003) Optical Properties of Metal Nanoparticles with Arbitrary Shapes. The Journal of Physical Chemistry B 107:6269-6275

49. Johnson PB and Christy RW (1972) Optical Constants of the Noble Metals. Physical Review B 6:4370-4379

50. Papavassiliou GC (1976) Surface plasmons in small Au-Ag alloy particles. Journal of Physics F: Metal Physics 6:L103-105

51. Tam F, Moran C and Halas N (2004) Geometrical Parameters Controlling Sensitivity of Nanoshell Plasmon Resonances to Changes in Dielectric Environment. The Journal of Physical Chemistry B 108:17290-17294

52. Prodan E, Lee A and Nordlander P (2002) The effect of a dielectric core and embedding medium on the polarizability of metallic nanoshells. Chemical Physics Letters 360:325332

53. Zhu J (2009) Composition-Dependent Plasmon Shift in Au-Ag Alloy Nanotubes: Effect of Local Field Distribution. The Journal of Physical Chemistry C 113:3164-3167

54. Chen JK, Zhu J, Li JJ and Zhao JW (2019) Switching the plasmon coupling of fractional hollow AuAg nanobox by asymmetrical etching of the inner Ag core. Journal of Physics D: Applied Physics 52:255301 
55. Zhu J (2005) Theoretical study of the optical absorption properties of $\mathrm{Au}-\mathrm{Ag}$ bimetallic nanospheres. Physica E: Low-dimensional Systems and Nanostructures 27:296-301 
Figures

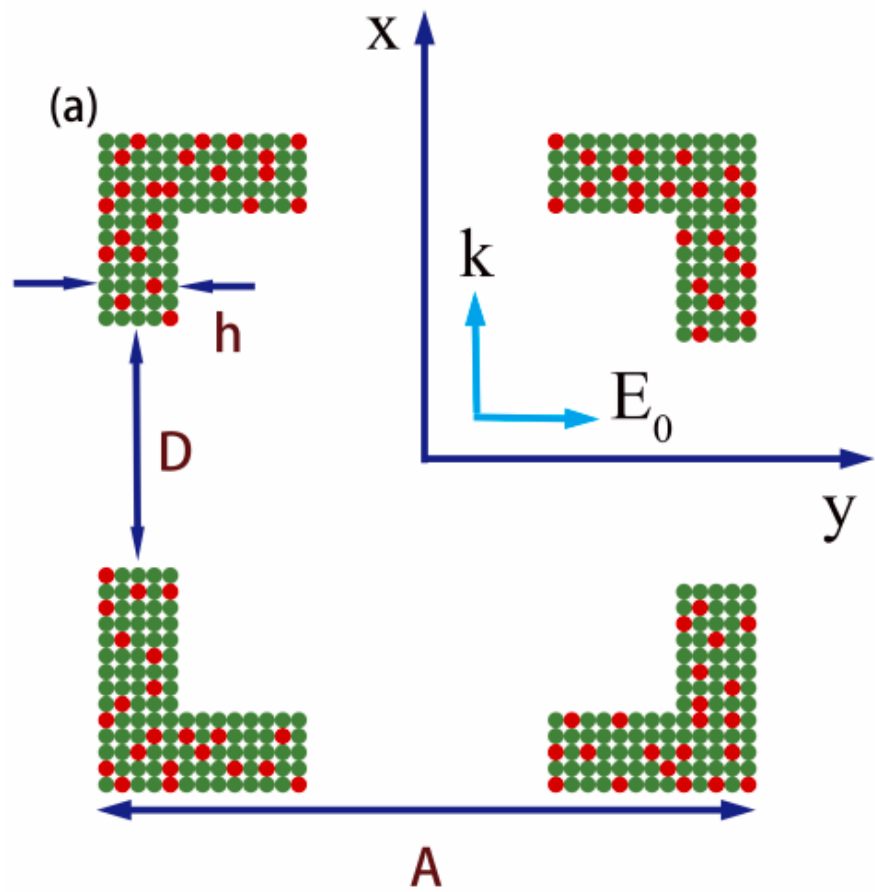

(b)
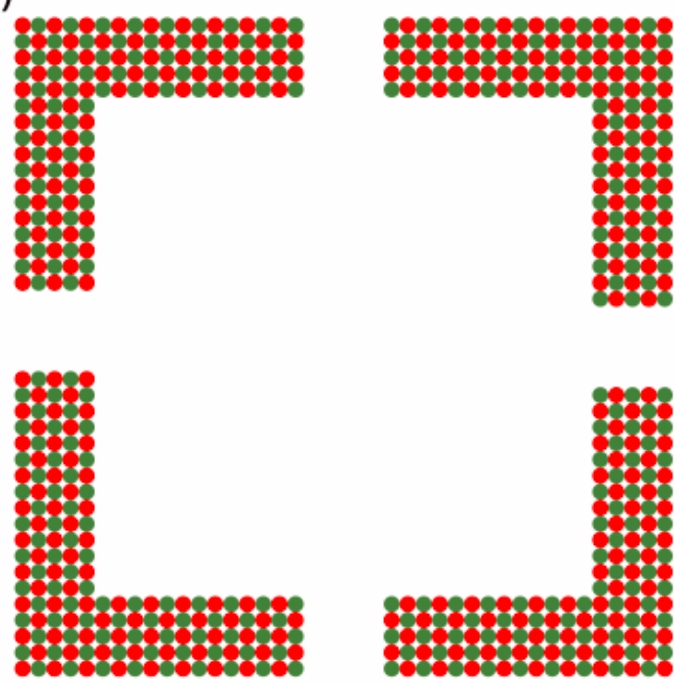

- $\mathrm{Au}$ atom $\quad \mathrm{Ag}$ atom

\section{Figure 1}

Please see the Manuscript PDF file for the complete figure caption 


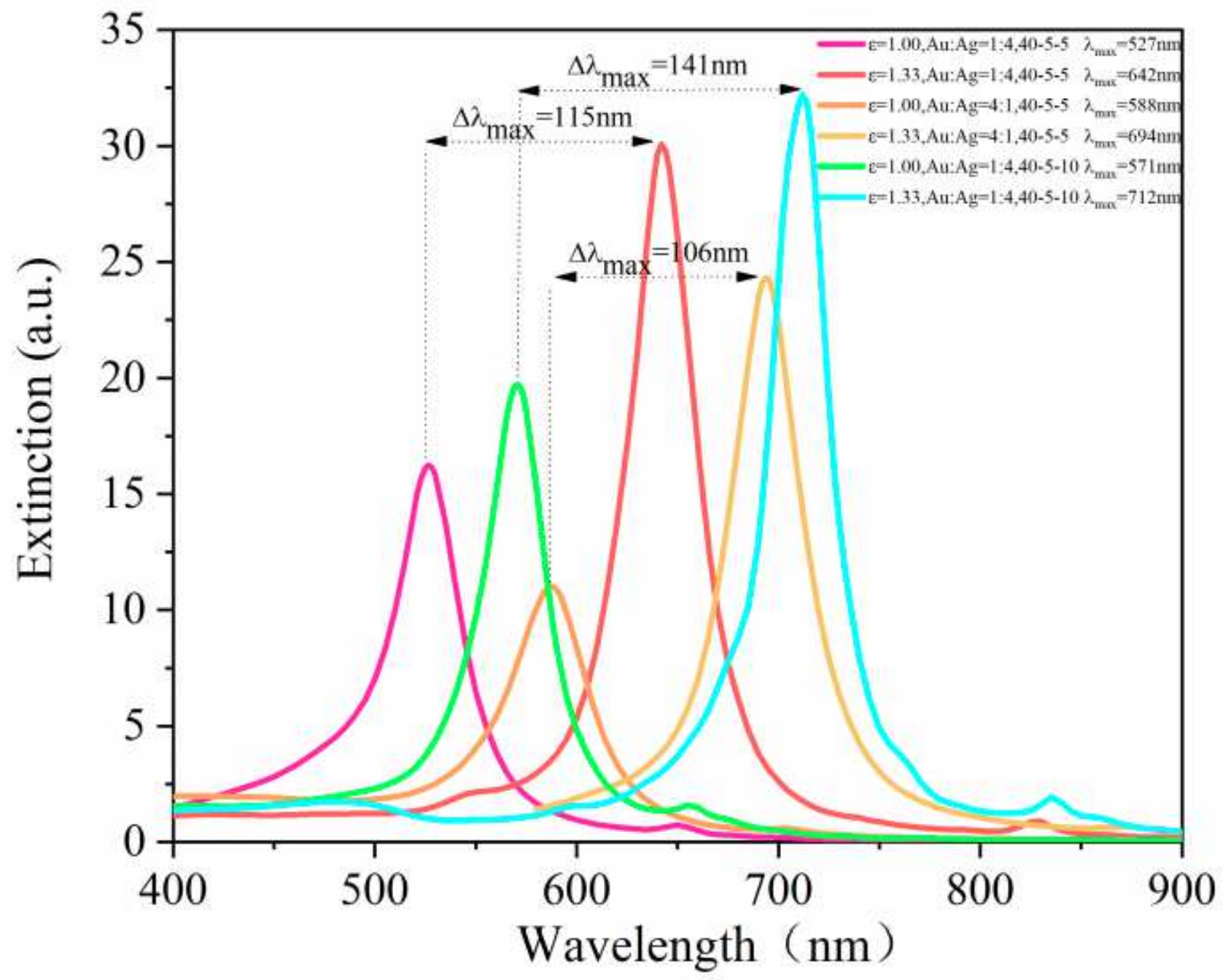

Figure 2

Please see the Manuscript PDF file for the complete figure caption 

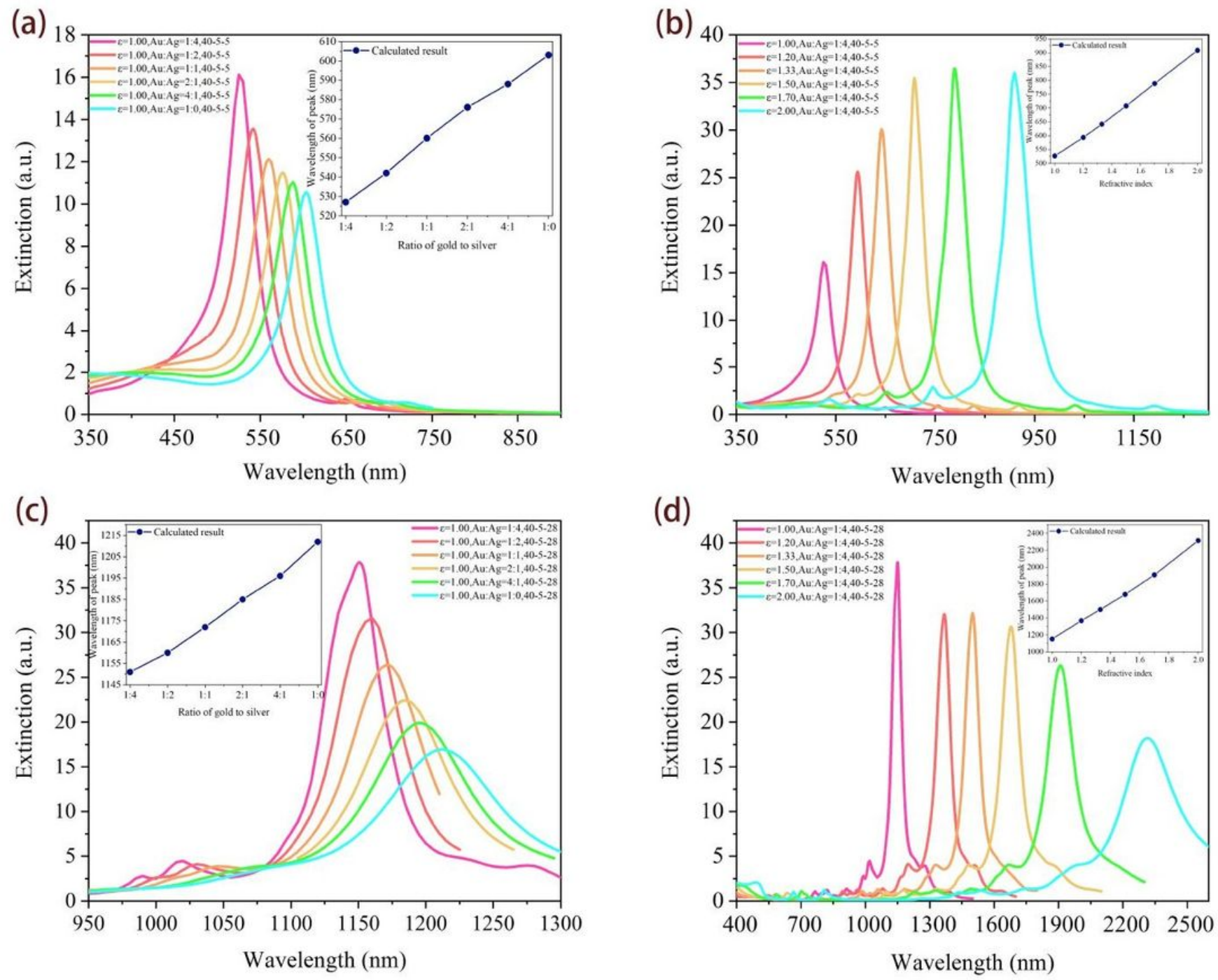

Figure 3

Please see the Manuscript PDF file for the complete figure caption 

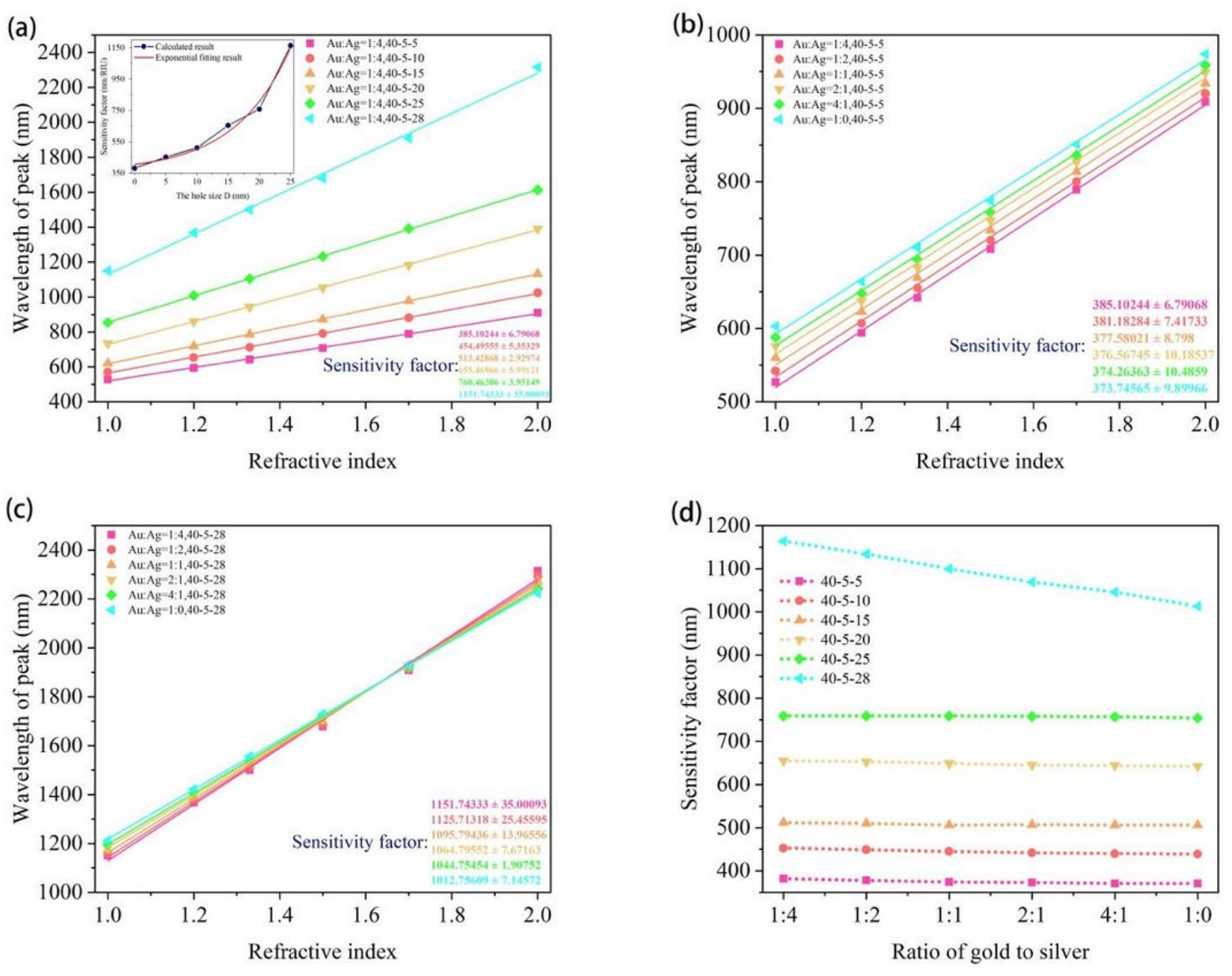

\section{Figure 4}

Please see the Manuscript PDF file for the complete figure caption
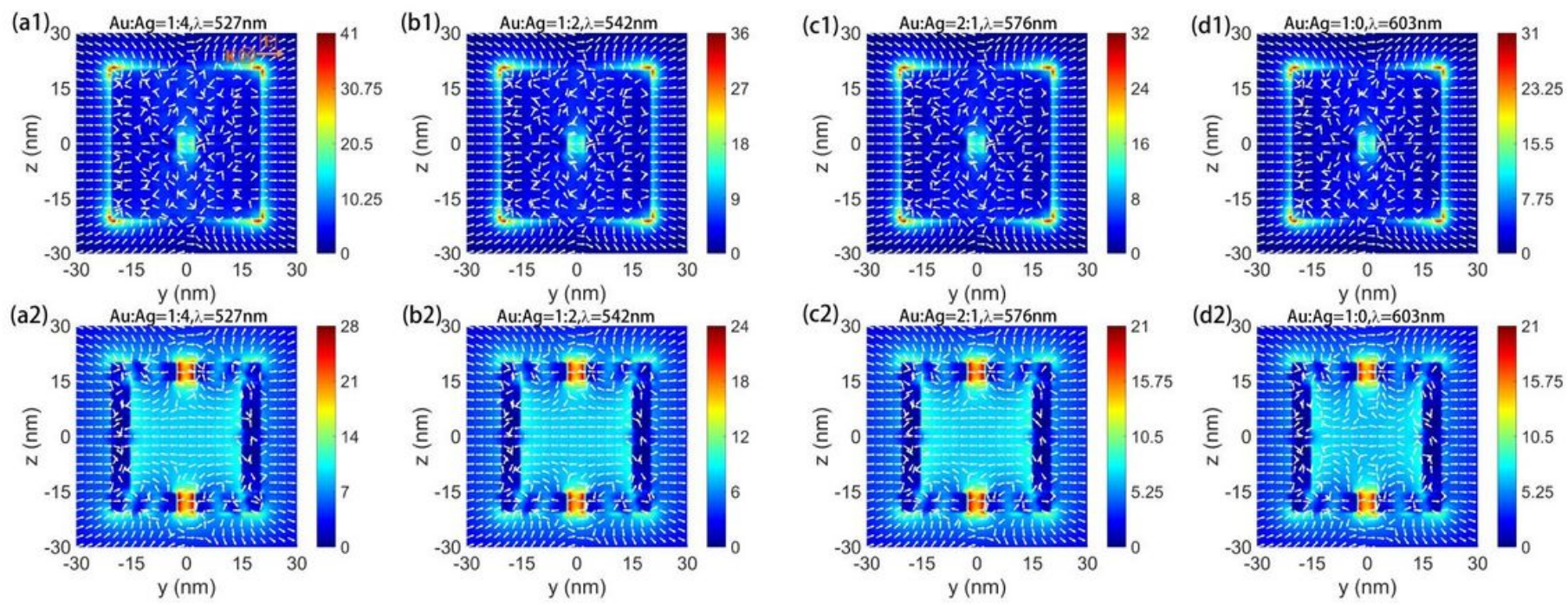
Figure 5

Please see the Manuscript PDF file for the complete figure caption
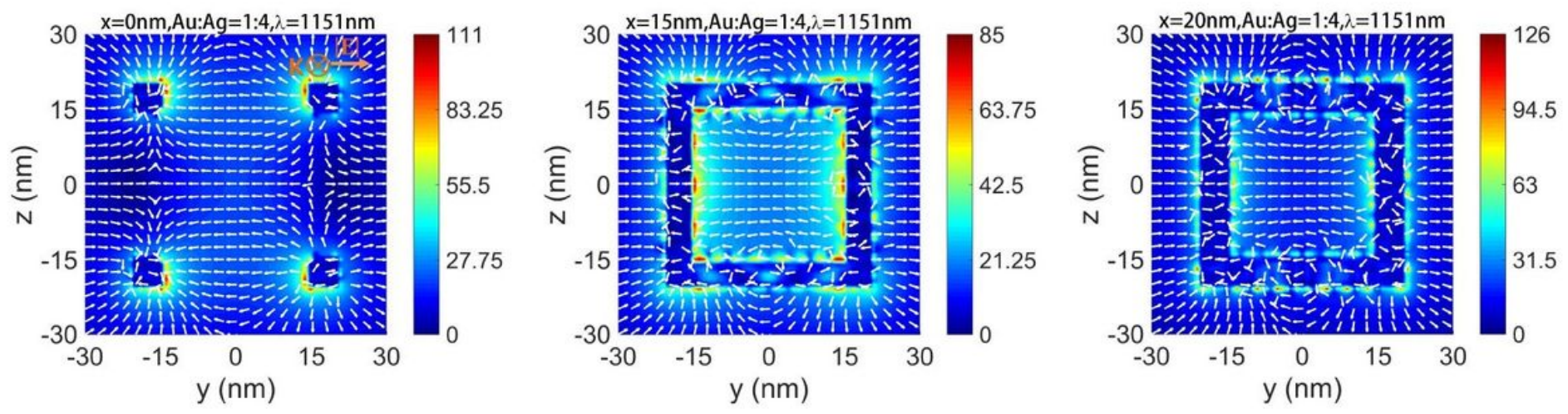

Figure 6

Please see the Manuscript PDF file for the complete figure caption
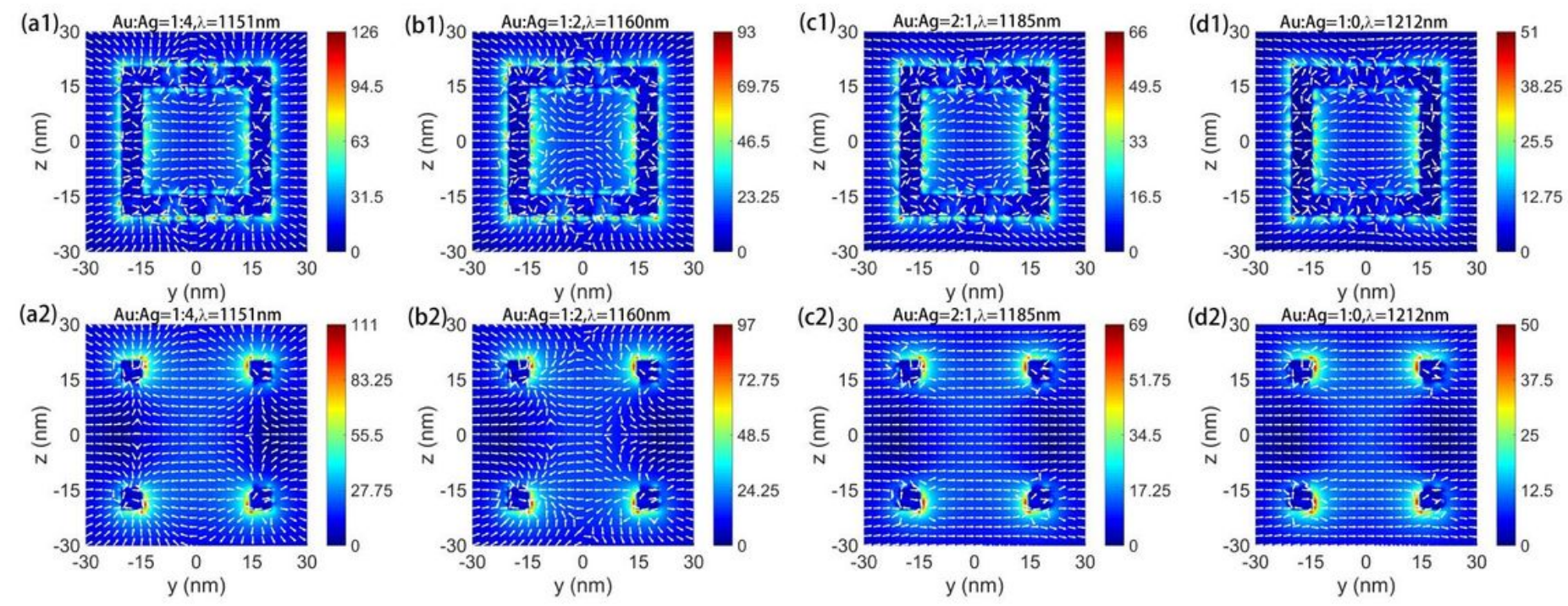

Figure 7

Please see the Manuscript PDF file for the complete figure caption 

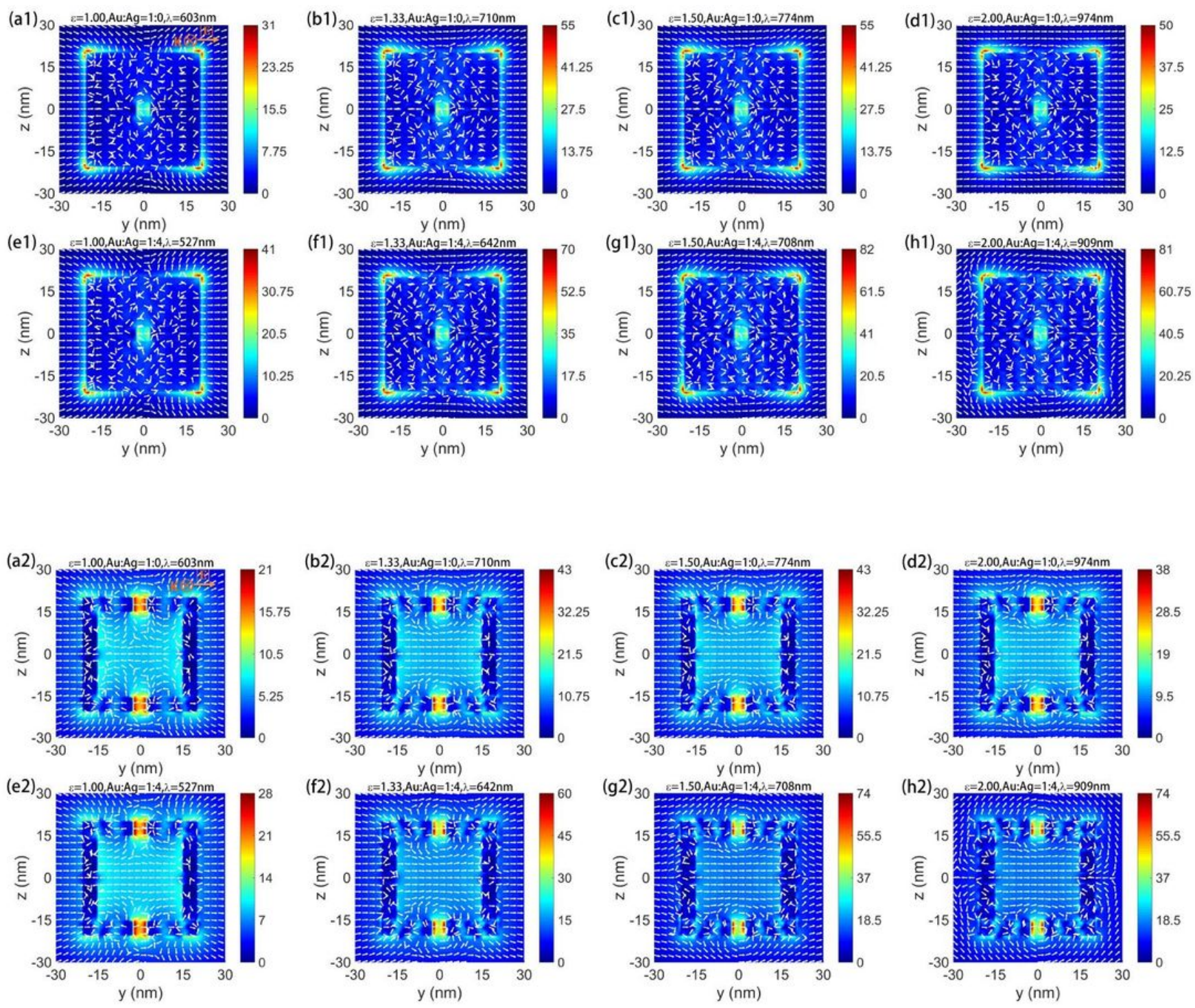

\section{Figure 8}

Please see the Manuscript PDF file for the complete figure caption 

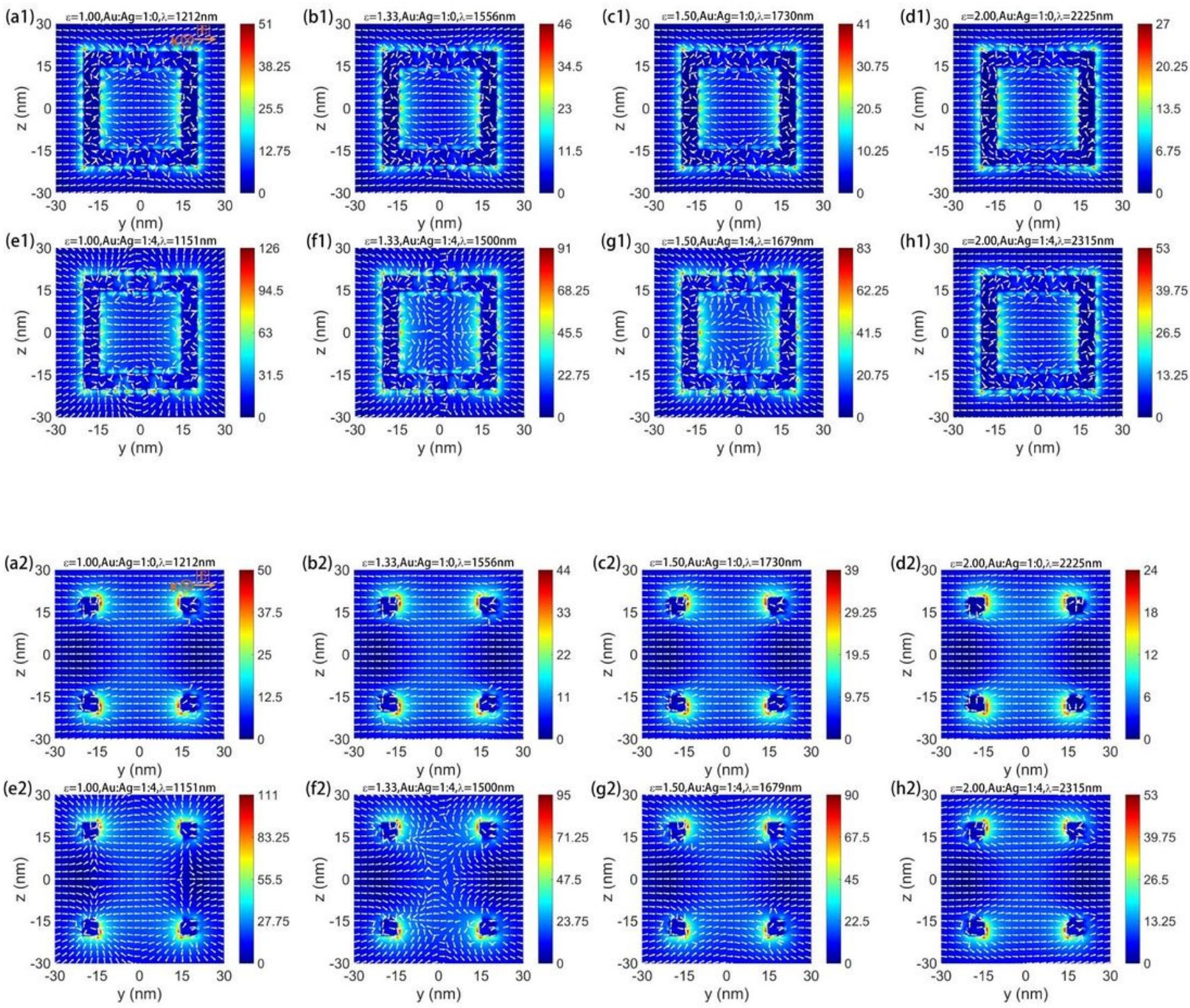

\section{Figure 9}

Please see the Manuscript PDF file for the complete figure caption 